\title{
Vector particle scattering on the lattice
}

\author{
F. Romero-López, A. Rusetsky, and C. Urbach \\ HISKP and BCTP, Rheinische Friedrich-Wilhelms Universität Bonn, 53115 Bonn, Germany
}

(Received 20 February 2018; published 6 July 2018)

\begin{abstract}
In this work, we present an explicit form of the Lüscher equation and consider the construction of the operators in different irreducible representations for the case of scattering of two vector particles. The formalism is applied to scalar QED in the Higgs Phase, where the $U(1)$ gauge boson acquires mass.
\end{abstract}

DOI: $10.1103 /$ PhysRevD.98.014503

\section{INTRODUCTION}

The study of scattering in Lattice Field Theory (LFT) starts with the original work of Lüscher [1]. In this work, an equation that relates the scattering phase shift of a spinless particle to the finite-volume spectrum in the rest frame was derived. The formalism has been extended to moving frames [2], $\pi-N$ scattering [3], $N-N$ scattering [4], different masses $[5,6]$, moving frames with different masses $[7,8]$ and any multichannel system with arbitrary spin, momentum and masses [9]. In practice, the extraction of the phase shifts from data in case of coupled channels is most conveniently done by the use of the $K$-matrix approach. This method was first proposed in Ref. [10], and a complete description can be found, e.g., in the recent work [11].

For the case of scattering of vector particles, there may be interesting issues that can be addressed through LFT, such as the possibility of the Higgs boson to be a bound state of two $W$ bosons. This is the case for a model proposed in Refs. $[12,13]$, where a "superstrong interaction" together with superstrongly interacting particles are present. Instead of the Higgs mechanism, a nonperturbative mass generation mechanism is suggested. The model is strongly coupled at the relevant scale, and, therefore, LFT is the approach to test whether this mechanism exists or not (see Refs. [14,15] for a first numerical investigation of this model). One possible consequence of this model could be that the Higgs represents a bound state in the $W W$ channel. This justifies a thorough study of the $W W$ interactions (including both the bound spectrum and scattering) within LFT, which is possible by using Lüscher's approach.

The aim of the present work is to study the vector-vector scattering process in a toy model, which is the first step towards applying the same method in physically more

Published by the American Physical Society under the terms of the Creative Commons Attribution 4.0 International license. Further distribution of this work must maintain attribution to the author(s) and the published article's title, journal citation, and DOI. Funded by SCOAP . interesting cases. To this end, we rederive the Lüscher equation for scattering of particles with arbitrary spin by using nonrelativistic effective theory and check that the results obtained are in agreement with Ref. [9]. We will further focus on the case of two identical vector particles and we make use of the spatial symmetries of the lattice to factorize the Lüscher equation. We explicitly construct the operators that transform under a certain irreducible representation of the spatial symmetry group, and, using these, we gain access to the different phase shifts of the theory. The approach will be tested in scalar QED, for which numerical results will be shown. For a first account of this work we refer to Ref. [16].

\section{SCATTERING OF TWO VECTOR PARTICLES}

\section{A. Derivation of Lüscher equation for arbitrary spin}

Let us consider a system of two particles with masses $m_{i}$, $i=1,2$ in $d=3$ dimensions. The system is described by the effective nonrelativistic Lagrangian

$\mathcal{L}=\phi_{1}^{\dagger} 2 W_{1}\left(i \partial_{t}-W_{1}\right) \phi_{1}+\phi_{2}^{\dagger} 2 W_{2}\left(i \partial_{t}-W_{2}\right) \phi_{2}+\mathcal{L}_{I}$.

Here, $\phi_{i}$ are the nonrelativistic fields with spin $s_{i}, W_{i}=$ $\left(m_{i}^{2}-\nabla^{2}\right)^{1 / 2}$ and the interactions are contained in $\mathcal{L}_{I}$. The corresponding nonrelativistic propagators, with $\omega_{i}(\mathbf{p})=$ $\left(m_{i}^{2}+\mathbf{p}^{2}\right)^{1 / 2}$, are diagonal in the spin indices $\nu, \nu^{\prime}$ :

$$
\begin{aligned}
\left(S_{i}(p)\right)_{\nu^{\prime} \nu} & =\frac{1}{2 \omega_{i}(\mathbf{p})} \frac{1}{\omega_{i}(\mathbf{p})-p^{0}-i \epsilon} \delta_{\nu^{\prime} \nu}, \\
\nu^{\prime}, \nu & =1, \ldots, 2 s_{i}+1 .
\end{aligned}
$$

The scattering T-matrix is defined through the LippmanSchwinger (LS) equation

$$
T(z)=\left(-H_{I}\right)+\left(-H_{I}\right)\left(-G_{0}(z)\right) T(z),
$$

where $H_{0}$ and $H_{I}$ are obtained from the Lagrangian in the usual way and $G_{0}(z)=\left(z-H_{0}\right)^{-1}$ is the free resolvent. The two-particle states with a total spin $S$ are given by 


$$
\left|\mathbf{k}_{\mathbf{1}}, \mathbf{k}_{\mathbf{2}}, S, \nu\right\rangle \equiv|\mathbf{P}, \mathbf{k}, S, \nu\rangle,
$$

with normalization

$$
\left\langle\mathbf{P}^{\prime}, \mathbf{k}^{\prime}, S^{\prime}, \nu^{\prime} \mid \mathbf{P}, \mathbf{k}, S, \nu\right\rangle=4 \omega_{1}\left(\mathbf{k}_{1}\right) \omega_{2}\left(\mathbf{k}_{\mathbf{2}}\right)(2 \pi)^{d} \delta^{d}\left(\mathbf{P}^{\prime}-\mathbf{P}\right)(2 \pi)^{d} \delta^{d}\left(\mathbf{k}^{\prime}-\mathbf{k}\right) \delta_{S^{\prime} S} \delta_{\nu^{\prime} \nu},
$$

where $\mathbf{k}_{\mathbf{1}}, \mathbf{k}_{\mathbf{2}}$ are the momenta of the particles, $S$ and $\nu$ denote the total spin and its projection for the two-particle system, respectively, and $\mathbf{P}, \mathbf{k}$ are the total and relative momenta in the laboratory frame:

$$
\mathbf{P}=\mathbf{k}_{\mathbf{1}}+\mathbf{k}_{\mathbf{2}}, \quad \mathbf{k}=\mu_{2} \mathbf{k}_{\mathbf{1}}-\mu_{1} \mathbf{k}_{\mathbf{2}}, \quad \mu_{1,2}=\frac{1}{2}\left(1 \pm \frac{m_{1}^{2}-m_{2}^{2}}{P^{2}}\right), \quad P_{0}=\omega_{1}\left(\mathbf{k}_{\mathbf{1}}\right)+\omega_{2}\left(\mathbf{k}_{\mathbf{2}}\right), \quad P^{2}=P_{0}^{2}-\mathbf{P}^{2} .
$$

Now define the matrix elements:

$$
\begin{aligned}
& t_{\nu^{\prime} \nu}^{S^{\prime} S}\left(\mathbf{k}^{\prime}, \mathbf{k}, \mathbf{P}, z\right)=\int \frac{d^{d} \mathbf{P}^{\prime}}{(2 \pi)^{d}}\left\langle\mathbf{P}^{\prime}, \mathbf{k}^{\prime}, S^{\prime}, \nu^{\prime}|T(z)| \mathbf{P}, \mathbf{k}, S, \nu\right\rangle, \\
& h_{\nu^{\prime} \nu}^{S^{\prime} S}\left(\mathbf{k}^{\prime}, \mathbf{k}, \mathbf{P}\right)=\int \frac{d^{d} \mathbf{P}^{\prime}}{(2 \pi)^{d}}\left\langle\mathbf{P}^{\prime}, \mathbf{k}^{\prime}, S^{\prime}, \nu^{\prime}\left|\left(-H_{I}\right)\right| \mathbf{P}, \mathbf{k}, S, \nu\right\rangle .
\end{aligned}
$$

One may rewrite the LS equation in terms of matrix elements, using Eqs. (7) and (8):

$$
t_{\nu^{\prime} \nu}^{S^{\prime} S}\left(\mathbf{k}^{\prime}, \mathbf{k}, \mathbf{P}, z\right)=h_{\nu^{\prime} \nu}^{S^{\prime} S}\left(\mathbf{k}^{\prime}, \mathbf{k}, \mathbf{P}\right)+\int \frac{d^{d} \mathbf{q}}{(2 \pi)^{d}} \sum_{S^{\prime \prime} \nu^{\prime \prime}} \frac{h_{\nu^{\prime} \nu^{\prime \prime}}^{S^{\prime} S^{\prime \prime}}\left(\mathbf{k}^{\prime}, \mathbf{q}, \mathbf{P}\right) t_{\nu^{\prime \prime} \nu}^{S^{\prime \prime} S}(\mathbf{q}, \mathbf{k}, \mathbf{P}, z)}{4 \omega_{1}\left(\mathbf{q}_{1}\right) \omega_{2}\left(\mathbf{P}-\mathbf{q}_{\mathbf{1}}\right)\left(\omega_{1}\left(\mathbf{q}_{\mathbf{1}}\right)+\omega_{2}\left(\mathbf{P}-\mathbf{q}_{\mathbf{1}}\right)-z\right)},
$$

where we define $\mathbf{q}=\mu_{2} \mathbf{q}_{1}-\mu_{1} \mathbf{q}_{2}$, as in Eq. (4). A key point here is that the elementary bubble (the free two-particle propagator, integrated over the relative momentum) is diagonal in spin, because also the single particle propagators are. However, the scattering amplitude need not be diagonal.

Now define the projectors to the partial waves in the CM frame, whose momenta are $\mathbf{k}^{*}$ :

$$
\Pi_{\nu^{\prime} \nu}^{A^{\prime} A}\left(\mathbf{k}^{\prime *}, \mathbf{k}^{*}\right)=\sum_{\rho, \rho^{\prime}} U_{\nu^{\prime} \rho^{\prime}}^{\left(S^{\prime}\right)}\left(\mathbf{k}^{*}\right)^{*} U_{\nu \rho}^{(S)}\left(\mathbf{k}^{*}\right)\left(\mathcal{Y}_{J^{\prime} l^{\prime} S^{\prime} \mu^{\prime}}\left(\mathbf{k}^{\prime *}, \rho^{\prime}\right)\right)^{*} \mathcal{Y}_{J l S \mu}\left(\mathbf{k}^{*}, \rho\right)
$$

where $A=(J, l, S, \mu), A^{\prime}=\left(J^{\prime}, l^{\prime}, S^{\prime}, \mu^{\prime}\right)$ represent multi-indices and $U_{\nu \rho}^{(S)}\left(\mathbf{k}^{*}\right)$ is the unitary transformation of the spin indices under a boost. The spherical harmonics with spin are defined as

$$
\mathcal{Y}_{J l S \mu}(\mathbf{k}, \nu)=\sum_{m, \sigma}\langle l S m \sigma \mid J \mu\rangle|\mathbf{k}|^{l} Y_{l m}(\hat{\mathbf{k}}) \chi_{\sigma}^{S}(\nu) \equiv|\mathbf{k}|^{l} Y_{J l S \mu}(\hat{\mathbf{k}}, \nu), \quad \hat{\mathbf{k}}=\mathbf{k} /|\mathbf{k}|,
$$

where $Y_{l m}$ denote usual spherical harmonics.

Using the projectors, the quantities in Eqs. (7) and (8) can be expanded as

$$
\begin{aligned}
t_{\nu^{\prime} \nu}^{S^{\prime} S}\left(\mathbf{k}^{\prime}, \mathbf{k}, \mathbf{P}, z\right) & =4 \pi \sum_{J^{\prime} l^{\prime} \mu^{\prime}, J l \mu} \Pi_{\nu^{\prime} \nu}^{A^{\prime} A}\left(\mathbf{k}^{\prime *}, \mathbf{k}^{*}\right) t_{A^{\prime} A}\left(\left|\mathbf{k}^{\prime *}\right|,\left|\mathbf{k}^{*}\right|, \mathbf{P}, z\right), \\
h_{\nu^{\prime} \nu}^{S^{\prime} S}\left(\mathbf{k}^{\prime}, \mathbf{k}, \mathbf{P}\right) & =4 \pi \sum_{J^{\prime} l^{\prime} \mu^{\prime}, J l \mu} \Pi_{\nu^{\prime} \nu}^{A^{\prime} A}\left(\mathbf{k}^{\prime *}, \mathbf{k}^{*}\right) h_{A^{\prime} A}\left(\left|\mathbf{k}^{\prime *}\right|,\left|\mathbf{k}^{*}\right|, \mathbf{P}\right) .
\end{aligned}
$$

If the system is placed in a box of a size $L$, the momenta are quantized. The integral in the LS equation should be replaced by a sum:

$$
\int \frac{d^{d} \mathbf{q}}{(2 \pi)^{d}} \rightarrow \frac{1}{L^{3}} \sum_{\mathbf{q}}, \quad \mathbf{q}=\frac{2 \pi}{L}\left(\mathbf{n}-\mu_{1} \mathbf{d}\right), \quad \mathbf{n} \in \mathbb{Z}^{3},
$$

where $2 \pi \mathbf{d} / L=\mathbf{P}$. 
By plugging the Eqs. (12) and (13) into the finite volume equivalent of Eq. (9), one gets:

$$
t_{A^{\prime} A}(s, \mathbf{P})-h_{A^{\prime} A}(s, \mathbf{P})=\frac{k^{*}}{8 \pi \sqrt{s}} \sum_{B^{\prime}, B} h_{A^{\prime} B^{\prime}}(s, \mathbf{P})\left(\left(\mathbf{k}^{*}\right)^{l+l^{\prime}} i^{l-l^{\prime}} \delta_{S_{B^{\prime}} S_{B}} \mathcal{M}_{B^{\prime} B}(s, \mathbf{P})\right) t_{B A}(s, \mathbf{P}),
$$

with $s=P^{2}$ and $S_{B}$ being the spin of the multi-index $B$. Note that using dimensional regularization, one is able to rewrite the LS equation as an algebraic equation, involving only the on-shell quantities. Hence, the quantities $t_{A^{\prime} A}$ and $h_{A^{\prime} A}$ in Eq. (15) coincide with their counterparts from Eqs. (12) and (13) on shell, i.e.,

$$
\left|\mathbf{k}^{\prime *}\right|=\left|\mathbf{k}^{*}\right|=\frac{\lambda^{1 / 2}\left(s, m_{1}^{2}, m_{2}^{2}\right)}{2 \sqrt{s}}, \quad z=P_{0},
$$

where $\lambda$ denotes the triangle function.

Now, using unitarity of the transformation of the spin indices, one arrives at

$$
\mathcal{M}_{J^{\prime} l^{\prime} S^{\prime} \mu^{\prime}, J l S \mu}(s, \mathbf{P})=\frac{32 \pi^{2}}{\left|\mathbf{k}^{*}\right|} \frac{\sqrt{s}}{L^{3}} i^{l-l^{\prime}} \delta_{S^{\prime} S} \sum_{\nu} \sum_{\mathbf{q}} \frac{\left(Y_{J^{\prime} l^{\prime} S \mu^{\prime}}\left(\hat{\mathbf{q}}^{*}, \nu\right)\right)^{*} Y_{J l S \mu}\left(\hat{\mathbf{q}}^{*}, \nu\right)}{4 \omega_{1}\left(\mathbf{q}_{\mathbf{1}}\right) \omega_{2}\left(\mathbf{P}-\mathbf{q}_{\mathbf{1}}\right)\left(\omega_{1}\left(\mathbf{q}_{\mathbf{1}}\right)+\omega_{2}\left(\mathbf{P}-\mathbf{q}_{\mathbf{1}}\right)-P_{0}\right)} .
$$

This matrix can be related to its equivalent for scalar particles by using Eq. (11):

$$
\mathcal{M}_{J^{\prime} l^{\prime} S^{\prime} \mu^{\prime}, J l S \mu}=\delta_{S^{\prime} S} \sum_{m^{\prime}, m, \sigma}\left\langle l^{\prime} S m^{\prime} \sigma \mid J^{\prime} \mu^{\prime}\right\rangle\langle l S m \sigma \mid J \mu\rangle \mathcal{M}_{l^{\prime} m^{\prime}, l m}
$$

where we used the identity [5,17] (with $\mathbf{q}=\mathbf{q}_{1}-\mu_{1} \mathbf{P}$ )

$$
\frac{1}{4 \omega_{1} \omega_{2}\left(\omega_{1}+\omega_{2}-P_{0}\right)}=\frac{1}{2 P_{0}} \frac{1}{\mathbf{q}^{2}-\frac{(\mathbf{q} \mathbf{P})^{2}}{P_{0}^{2}}-\left(\mathbf{k}^{*}\right)^{2}}+\frac{1}{4 \omega_{1} \omega_{2}}\left(\frac{1}{\omega_{1}+\omega_{2}+P_{0}}-\frac{1}{\omega_{1}-\omega_{2}+P_{0}}-\frac{1}{\omega_{2}-\omega_{1}+P_{0}}\right),
$$

kept only the singular part [first term in Eq. (19)] and used $\left(\mathbf{q}^{*}\right)^{2}=\mathbf{q}^{2}-\frac{(\mathbf{q} \mathbf{P})^{2}}{P_{0}^{2}}$. This way, and up to exponentially suppressed terms, $\mathcal{M}_{l^{\prime} m^{\prime}, l m}$ is given by (see Ref. [5])

$$
\mathcal{M}_{l^{\prime} m^{\prime}, l m}\left(\mathbf{k}^{*}, s\right)=\frac{(-1)^{l^{\prime}}}{\pi^{3 / 2} \gamma} \sum_{j=\left|l-l^{\prime}\right|}^{l+l^{\prime}} \sum_{s=-j}^{j} \frac{i^{j}}{\eta^{j+1}} Z_{j s}^{d}(1, s)^{*} C_{l^{\prime} m^{\prime}, j s, l m}, \quad \eta=\frac{\left|\mathbf{k}^{*}\right| L}{2 \pi},
$$

where

$$
\begin{gathered}
C_{l^{\prime} m^{\prime}, j s, l m}=(-1)^{m} i^{l^{\prime}-j+l} \sqrt{(2 l+1)\left(2 l^{\prime}+1\right)(2 j+1)}\left(\begin{array}{ccc}
l^{\prime} & j & l \\
m^{\prime} & s & -m
\end{array}\right)\left(\begin{array}{ccc}
l^{\prime} & j & l \\
0 & 0 & 0
\end{array}\right), \\
Z_{l m}^{d}(1, s)=\sum_{\mathbf{r} \in P_{d}} \frac{|\mathbf{r}|^{l} Y_{l m}(r)}{\mathbf{r}^{2}-\eta^{2}}, \quad P_{d}=\left\{\mathbf{r}_{\|}=\gamma^{-1}\left(\mathbf{n}_{\|}-\mu_{1} \mathbf{d}\right), \mathbf{r}_{\perp}=\mathbf{n}_{\perp}\right\},
\end{gathered}
$$

$\gamma=\left(1-\mathbf{P}^{2} / P_{0}^{2}\right)^{-1 / 2}$ and $\mathbf{n} \in \mathbb{Z}^{3}$. One can see that Eq. (15) is a matrix equation, and the poles in $t_{A^{\prime} A}$ arise when

$$
\operatorname{det} \mathcal{A}=0 \text {, }
$$

where $\mathcal{A}$ is a matrix

$$
\mathcal{A}_{J^{\prime} l^{\prime} S^{\prime} \mu^{\prime}, J l S \mu}=\frac{8 \pi \sqrt{s}}{\left|\mathbf{k}^{*}\right|^{l+l^{\prime}+1}}\left(h_{l^{\prime} S^{\prime}, l S}^{J}\right)^{-1} \delta_{J^{\prime} J} \delta_{\mu^{\prime} \mu}-\delta_{S^{\prime} S} \mathcal{M}_{J^{\prime} l^{\prime} S \mu^{\prime}, J l S \mu}
$$

Here it is already implied that $J$ and $\mu$ are conserved in scattering processes in the infinite volume, i.e., $h_{J^{\prime} l^{\prime} S^{\prime} \mu^{\prime}, J l S \mu}=h_{l^{\prime} S^{\prime}, l S^{\prime}}^{J} \delta_{J^{\prime} J} \delta_{\mu^{\prime} \mu}$, and the factor $i^{l-l^{\prime}}$ can be dropped in the determinant. 
TABLE I. Possible values of $J^{P}$ with $J<3$.

\begin{tabular}{lcccccc}
\hline \hline$J^{P}$ & $0^{+}$ & $0^{-}$ & $1^{+}$ & $1^{-}$ & $2^{+}$ & $2^{-}$ \\
\hline$\{S, L\}$ & $\{0,0\}$ & \multirow{2}{*}{1,1} & & & $\{0,2\}$ & $\{1,1\},\{1,3\}$ \\
\hline \hline
\end{tabular}

Now, in order to express this equation in a more compact way, one uses the standard definition of the $S$ matrix (see [18], for nucleon-nucleon scattering), $S=e^{2 i \delta(s)}$, in terms of the phase shift $\delta(s)$. This way, one can write down $h_{l^{\prime} S^{\prime}, l S}^{J}$ in terms of $\delta$ :

$$
h_{l^{\prime} S^{\prime}, l S}^{J}=\frac{8 \pi \sqrt{s}}{\left|\mathbf{k}^{*}\right|^{l+l^{\prime}+1}}(\tan \delta)_{l^{\prime} S^{\prime}, l S^{\prime}}^{J}
$$

Plugging it in Eq. (24), we arrive at

$$
\mathcal{A}_{J^{\prime} l^{\prime} S^{\prime} \mu^{\prime}, J l S \mu}=(\cot \delta)_{l^{\prime} S^{\prime}, l S}^{J} \delta_{J^{\prime} J} \delta_{\mu^{\prime} \mu}-\delta_{S^{\prime} S} \mathcal{M}_{J^{\prime} l^{\prime} S \mu^{\prime}, J l S \mu} .
$$

\section{B. Two vector particles}

A system of two identical vector particles can couple to total spin $S=0,1,2$. Even spin combinations are symmetric under the exchange of two particles, whereas odd combinations are antisymmetric. The same holds for the angular momentum $L$. The possible combinations of $S$ and $L$ to $J^{P}$, respecting Bose statistics (totally symmetric state), are listed in the Table I. The combinations that have mixing are in the same column in the table and correspond to same $J^{P}$ but different $L, S$. The possible mixings can be parametrized by a mixing angle and two eigenvalues. This would be analogous to the parametrization of the mixings for two nucleons in Ref. [18]; for example:

$$
\cot \delta^{0^{+}}=\left(\begin{array}{cc}
\cos \epsilon_{0} & -\sin \epsilon_{0} \\
\sin \epsilon_{0} & \cos \epsilon_{0}
\end{array}\right)\left(\begin{array}{cc}
\cot \delta_{1}^{0^{+}} & 0 \\
0 & \cot \delta_{2}^{0^{+}}
\end{array}\right)\left(\begin{array}{cc}
\cos \epsilon_{0} & \sin \epsilon_{0} \\
-\sin \epsilon_{0} & \cos \epsilon_{0}
\end{array}\right) .
$$

Since no mixing occurs between even and odd spins, neither in the $\mathcal{M}$ matrix, nor in the phase shifts, Eq. (26) factorizes for even and odd spin.

\section{Effective range expansion in case of multiple channels}

For the scattering of two spinless particles, it is well known (see Ref. [19]) that the phase shift can be parametrized as a polynomial of $\mathbf{k}^{2}$ :

$$
\mathbf{k}^{2 l+1} \cot \delta_{l}=\sum_{n=0} a_{n l} \mathbf{k}^{2 n}
$$

One obviously needs an analog of this parametrization in the multichannel case as well. ${ }^{1}$ In order to derive such a parametrization, we note that, within the effective field theory, the left-hand side of Eq. (13) has a Taylor expansion in momenta. Taking now into account the fact that the projector on the right-hand side of the same equation contains the factor $\left|\mathbf{k}^{*}\right|^{l+l^{\prime}}$, from Eq. (25) one may finally conclude that, on the mass shell,

$$
\mathbf{k}^{l+l^{\prime}+1} \cot \delta_{l^{\prime} S^{\prime}, l S}^{J}=\sum_{n=0}\left(a_{n}\right)_{l^{\prime} S^{\prime}, l S} \mathbf{k}^{2 n}
$$

\footnotetext{
${ }^{1}$ An equivalent derivation can be found in Refs. [20,11].
}

\section{Reduction of the Lüscher equation}

Our aim here is to construct the basis vectors of all irreducible representations (irreps) from the basis vectors of the irreps of the rotation group, corresponding to the symmetry in the infinite volume, and to (partially) diagonalize the Lüscher equation in this new basis. The general procedure is well known in the literature, so we shall skip many details ${ }^{2}$ Let $\mathcal{G}$ be a full octahedral group including inversions, or a subgroup thereof (little group), which is the symmetry group in the moving frames. Let $\Gamma$ be a certain irrep of $\mathcal{G}$, and let $\alpha=1, \ldots \operatorname{dim} \Gamma$ be an index labeling basis vector in this representation. One can construct these basis vectors by applying certain projection operators to the basis vectors of the irreps of the rotation group. These (unnormalized) projectors are given by

$$
\left(P_{\alpha \beta}^{\Gamma, J, l}\right)_{\mu \mu^{\prime}}=\sum_{\mathcal{S} \in G}\left(R_{\alpha \beta}^{\Gamma}(\mathcal{S})\right)^{*} D_{\mu \mu^{\prime}}^{J}(\mathcal{S})
$$

Here, $D_{\mu \mu^{\prime}}^{J}(\mathcal{S})$ denotes the usual Wigner matrix, if $\mathcal{S}$ corresponds to a pure rotation. Otherwise, the group elements can be represented as $\mathcal{S}=I \overline{\mathcal{S}}$, where $I$ is an inversion and $\overline{\mathcal{S}}$ is a pure rotation. In this case, we define

\footnotetext{
${ }^{2}$ See, for example, Ref. [21], where the same problem has been considered by using the helicity formalism.
} 
$D_{\mu \mu^{\prime}}^{J}(\mathcal{S})=(-1)^{l} D_{\mu \mu^{\prime}}^{J}(\overline{\mathcal{S}})$. Furthermore, $R_{\alpha \beta}^{\Gamma}(\mathcal{S})$ denotes a matrix representation of $\mathcal{G}$ in the irrep $\Gamma$.

These projectors must be applied to the basis vectors of the irreps of the rotation group $|J, S, l, \mu\rangle$ with indices $\beta$ and $\mu$ fixed

$$
|\Gamma, \alpha, J, S, l, n\rangle \propto \sum_{\mu^{\prime}}\left(P_{\alpha \beta}^{\Gamma, J, l}\right)_{\mu \mu^{\prime}}\left|J, S, l, \mu^{\prime}\right\rangle,
$$

where $n$ labels the number of multiple occurrences of $\Gamma$. The different spatial symmetry groups, with their irreducible representations and the corresponding elements are listed in Appendices A and B.

As seen from Eq. (31), the basis vectors of the irreducible representations of the symmetry group of the lattice can be expressed in terms of the one of the continuum:

$$
|\Gamma, \alpha, J, l, S, n\rangle=\sum_{\mu} c_{J l \mu}^{\Gamma n \alpha}|J l S \mu\rangle,
$$

where the Clebsch-Gordan coefficients $c_{J l \mu}^{\Gamma n \alpha}$ can be read from Tables X-XVII in Appendix C. They are in agreement with those of Refs. [3,8] and obey the usual orthogonality conditions

$$
\sum_{\mu}\left(c_{J l \mu}^{\Gamma^{\prime} n^{\prime} \alpha^{\prime}}\right)^{*} c_{J l \mu}^{\Gamma n \alpha}=\delta_{\Gamma^{\prime} \Gamma} \delta_{\alpha^{\prime} \alpha} \delta_{n^{\prime} n}
$$

The matrix $\mathcal{M}$ can be partially diagonalized in the new basis:

$$
\left\langle\Gamma^{\prime}, \alpha^{\prime}, J^{\prime}, l^{\prime}, S, n^{\prime}|\mathcal{M}| \Gamma, \alpha, J, l, S, n\right\rangle=\mathcal{M}_{J^{\prime} l^{\prime} S n^{\prime}, J l S n}^{\Gamma} \delta_{\Gamma^{\prime} \Gamma} \delta_{\alpha^{\prime} \alpha},
$$

where

$$
\mathcal{M}_{J^{\prime} l^{\prime} S n^{\prime}, J l S n}^{\Gamma}=\sum_{\mu \mu^{\prime}}\left(c_{J^{\prime} l^{\prime} \mu^{\prime}}^{\Gamma n^{\prime} \alpha}\right)^{*} c_{J l \mu}^{\Gamma n \alpha} \mathcal{M}_{J^{\prime} l^{\prime} S \mu^{\prime}, J L S \mu}
$$

(for a given $\Gamma$ and $\alpha$ ). Moreover, the matrix $\cot \delta$ should be written down in the same basis as $\mathcal{M}$ :

$$
\begin{aligned}
(\cot \delta)_{J^{\prime} l^{\prime} S^{\prime} n^{\prime}, J l S n}^{\Gamma} & =\sum_{\mu^{\prime} \mu}\left(c_{J^{\prime} l^{\prime} \mu^{\prime}}^{\Gamma n^{\prime} \alpha} c_{J l \mu}^{*} c_{J n \alpha}^{\Gamma n \alpha}(\cot \delta)_{l^{\prime} S^{\prime}, l S}^{J} \delta_{\mu \mu^{\prime}} \delta_{J J^{\prime}}\right. \\
& =\delta_{J J^{\prime}} \delta_{n n^{\prime}}(\cot \delta)_{l^{\prime} S^{\prime}, l S}^{J} .
\end{aligned}
$$

Here, we have used Eq. (33) and the fact that only states with the same parity can mix. Now one sees that the determinant factorizes:

$$
\prod_{S=\text { even }} \prod_{\Gamma} \operatorname{det} \mathcal{A}^{\Gamma}=0
$$

where

$\mathcal{A}_{J^{\prime} l^{\prime} S^{\prime} n^{\prime}, J L S n}^{\Gamma}=(\cot \delta)_{l^{\prime} S^{\prime}, l S}^{J} \delta_{J J^{\prime}} \delta_{n n^{\prime}}-\delta_{S S^{\prime}} \mathcal{M}_{J^{\prime} l^{\prime} S n^{\prime}, J l S n}^{\Gamma}$

For simplicity, $\mathcal{M}^{\Gamma}$ will be expressed in terms of functions $\omega_{j s}$

$$
\omega_{j s}=\frac{\gamma^{-1} \eta^{-j-1}}{\pi^{3 / 2} \sqrt{2 j+1}} Z_{j s}^{d}(1, s) .
$$

Understanding and using some symmetry properties of $\omega_{l m}$ simplifies the expressions of $\mathcal{M}^{\Gamma}$. A frame-independent property is

$$
Z_{l m}^{d}=(-1)^{m} Z_{l-m}^{*},
$$

and it is a direct consequence of the properties of the spherical harmonics. Moreover, if one changes the order of the particles $\left(m_{1} \leftrightarrow m_{2}\right)$ :

$$
Z_{j s}^{d,\left(m_{1}, m_{2}\right)}=(-1)^{j} Z_{j s}^{d,\left(m_{2}, m_{1}\right)},
$$

which for the case of equal masses implies vanishing $Z_{j s}^{d}$ for odd $\mathrm{j}$. Additional symmetry properties of $\omega_{j s}$ in the rest frame are listed in Table II for the rest frame (See also [22].) and in Ref. [8] for moving frames. In Appendix E we give some examples of $\mathcal{M}^{\Gamma}$ in the rest frame.

TABLE II. Possible values of $\omega_{l m}$ in the rest frame.

\begin{tabular}{lc}
\hline \hline$l$ & $\omega_{l}$ \\
\hline 0 & $\left(\omega_{00}\right)$ \\
1 & $(0,0,0)$ \\
2 & $(0,0,0,0,0)$ \\
3 & $(0,0,0,0,0,0,0)$ \\
4 & $\left(\frac{5}{\sqrt{70}} \omega_{40}, 0,0,0, \omega_{40}, 0,0,0, \frac{5}{\sqrt{70}} \omega_{40}\right)$ \\
5 & $(0,0,0,0,0,0,0,0,0,0,0)$ \\
6 & $\left(0,0,-\sqrt{\frac{7}{2}} \omega_{60}, 0,0,0, \omega_{60}, 0,0,0,-\sqrt{\frac{7}{2}} \omega_{60}, 0,0\right)$ \\
7 & $(0,0,0,0,0,0,0,0,0,0,0,0,0,0,0)$ \\
8 & $\left(\sqrt{\frac{65}{198}} \omega_{80}, 0,0,0, \sqrt{\frac{14}{99}} \omega_{80}, 0,0,0, \omega_{80}, 0,0,0, \sqrt{\frac{14}{99}} \omega_{80}, 0,0,0, \sqrt{\frac{65}{198}} \omega_{80}\right)$ \\
\hline \hline
\end{tabular}




\section{TOY MODEL: SCALAR QED}

\section{A. The Lagrangian}

In order to test the formalism, we use scalar QED with a Higgs mechanism, since the vector state needs to be massive. This model was, for instance, studied in Ref. [23], whose parameters will be used as a guideline.
The continuum Euclidean Lagrangian of such a theory reads

$\mathcal{L}_{E}=\frac{1}{4} F^{\mu \nu} F_{\mu \nu}+\left(D_{\mu} \phi_{c}\right)^{\dagger} D_{\mu} \phi_{c}+m_{0}^{2}\left|\phi_{c}\right|^{2}+\lambda_{c}\left|\phi_{c}\right|^{4}$,

with $D_{\mu} \phi_{c}=\partial_{\mu} \phi_{c}+i g A_{\mu} \phi_{c}$ and $F_{\mu \nu}=\partial_{\mu} A_{\nu}-\partial_{\nu} A_{\mu}$.

For the discretization we restrict space-time to a discrete and finite set of points

$$
x \in\left\{\left(x_{0}, x_{1}, x_{2}, x_{3}\right) \mid x_{0}=0,1, \ldots, T, x_{i}=0,1, \ldots, L-1, i=1,2,3\right\} .
$$

We use periodic boundary conditions. In order to include the gauge symmetry in the discretized action, one defines the discretized gauge links at point $x$ in direction $\mu$ as

$$
U_{x, \mu}=e^{i a g A_{x, \mu},}
$$

with gauge potential $A_{x, \mu}$, gauge coupling $g$ and lattice spacing $a$. In the case of QED $U_{x, \mu} \in U(1)$. Scalar field $\phi$, covariant derivative and integrals are discretized as follows:

$$
\begin{aligned}
a \phi_{c}(x) & \rightarrow \sqrt{\kappa} \phi_{x}, \\
D_{\mu} \phi_{c}(x) & \rightarrow \kappa\left(U_{x, \mu} \phi_{x+\mu}-\phi_{x}\right) / a^{2}, \\
\int d^{4} x & \rightarrow a^{4} \sum_{x} .
\end{aligned}
$$

This way, the discretized action reads

$$
S=\sum_{x}\left(-\frac{\beta}{2} \sum_{\mu<\nu}\left(U_{x, \mu \nu}+U_{x, \mu \nu}^{*}\right)-\kappa \sum_{\mu}\left(\phi_{x}^{*} U_{x, \mu} \phi_{x+\mu}+c c\right)+\lambda\left(\left|\phi_{x}\right|^{2}-1\right)^{2}+\left|\phi_{x}\right|^{2}\right),
$$

with

$$
\lambda_{c}=\frac{\lambda}{\kappa^{2}}, \quad\left(a m_{0}\right)^{2}=\frac{1-2 \lambda-8 \kappa}{\kappa}, \quad \beta=\frac{1}{g^{2}} .
$$

The plaquette at point $x$ in the $\mu-\nu$ plane is defined as usual by the smallest closed loop

$$
U_{x, \mu \nu}=U_{x, \mu} U_{x+\mu, \nu} U_{x, \nu}^{\dagger} U_{x+\nu, \mu}^{\dagger} .
$$

\section{B. Construction of the operators}

Any transformation of the group $\mathcal{G}$, acting on the components of any vector, is a combination of an interchange of its components, an inversion of an axis and an inversion of all axes. We would like to study the transformation properties of the operators

$$
\mathcal{O}_{i}(x)=\phi_{x}^{\dagger} U_{x, i} \phi_{x+i}
$$

with respect to the transformations from the group $\mathcal{G}$ (here, we choose the spatial component $\mu=i$ of the link $\left.U_{x, \mu}\right)$. We will consider everything in the continuum first and then its equivalent for the discretized model.
The transformation of the scalar fields reads

$$
\phi(x) \rightarrow \phi\left(x^{\prime}\right), \quad x_{i}^{\prime}=T_{i j}\left(\mathcal{S}^{-1}\right) x_{j}, \quad t^{\prime}=t,
$$

where the matrices $T_{i j}\left(\mathcal{S}^{-1}\right)$ form a three-dimensional irrep of the cubic group in the Cartesian basis. Next, we consider the transformation of the link. The transformation law for the vector field is given by

$$
A_{i}(x) \rightarrow T_{i j}\left(\mathcal{S}^{-1}\right) A_{j}\left(x^{\prime}\right) .
$$

For the transformation of a link under $\mathcal{G}$ multiple cases have to be taken into account:

(i) The interchange of the components does not affect the index $i$. For example, $i=1$, whereas the components 2,3 are interchanged. Then, in the continuum, the link transforms as

$$
\begin{aligned}
U\left(x, x+a e_{1}\right) & \rightarrow \exp \left(i g \int_{0}^{1} d \tau a A_{1}\left(x_{1}+a \tau, x_{3}, x_{2}, t\right)\right) \\
& =U\left(x^{\prime}, x^{\prime}+a e_{1}\right),
\end{aligned}
$$

where $e_{i}$ denotes a unit vector in the direction $i$ and $x^{\prime}=\left(x_{1}, x_{3}, x_{2}, t\right)$. On the lattice, this corresponds to

$$
U_{x, i} \rightarrow U_{x^{\prime}, i} .
$$


(ii) The interchange involves the component $i$, e.g., $i=1$ and the components 1,2 are interchanged. Then,

$$
\begin{aligned}
U\left(x, x+a e_{1}\right) & \rightarrow \exp \left(i g \int_{0}^{1} d \tau a A_{2}\left(x_{2}, x_{1}+a \tau, x_{3}, t\right)\right) \\
& =U\left(x^{\prime}, x^{\prime}+a e_{2}\right),
\end{aligned}
$$

or, on the lattice,

$$
U_{x, i} \rightarrow U_{x^{\prime}, j}
$$

where $x^{\prime}$ is obtained from $x$ by interchanging the components $x_{i}$ and $x_{j}$.

Both transformations can be written as

$$
U_{x, i} \rightarrow T_{i j}\left(\mathcal{S}^{-1}\right) U_{x^{\prime}, j}, \quad x_{i}^{\prime}=T_{i j}\left(\mathcal{S}^{-1}\right) x_{j} .
$$

In other words, the link $U_{x, i}$ behaves like a vector under such transformations, albeit not being a vector with respect to the rotation group.

(iii) $i=1$ and the reflection of all axes. The result is given by

$$
U\left(x, x+a e_{1}\right) \rightarrow \exp \left(-i g \int_{0}^{1} d \tau a A_{1}\left(-x_{1}-a \tau,-x_{2},-x_{3}, t\right)\right)=U^{\dagger}\left(x^{\prime}-a e_{1}, x^{\prime}\right)
$$

or, on the lattice,

$$
U_{x, i} \rightarrow U_{x^{\prime}-i, i}^{\dagger} .
$$

(iv) The inversion of one of the axes. Here, again, one has to consider two different possibilities. First, if the axis $i$ is not affected by inversion, then

$$
U_{x, i} \rightarrow U_{x^{\prime}, i}
$$

otherwise

$$
U_{x, i} \rightarrow U_{x^{\prime}-i, i}^{\dagger}
$$

Finally, let us consider the set of the operators $\mathcal{O}_{i}(x)$, defined in Eq. (48) and construct the operators

$$
\overline{\mathcal{O}}_{i}(x)=\phi_{x}^{\dagger} U_{x-i, i}^{\dagger} \phi_{x-i} .
$$

Using the transformation properties of the scalar field and a link it is straightforward to check that the following operator

$$
S(x)=\sum_{i}\left(\mathcal{O}_{i}(x)+\overline{\mathcal{O}}_{i}(x)\right)
$$

transforms as $S(x) \rightarrow S\left(x^{\prime}\right)$ both under rotations and inversions. One may use this operator, for example, to project out the spectrum in the representation $A_{1}$ (rest frame).

On the other hand, it can be checked that the operator

$$
V_{i}(x)=\mathcal{O}_{i}(x)-\overline{\mathcal{O}}_{i}(x)
$$

behaves like a vector both under the rotations and reflections. We shall use this operator to construct the two-particle operators for the vector-vector scattering in different irreps. The conventions and the naming scheme of these irreps are listed in Appendix A.

The generalization for the case of a Wilson line of arbitrary length is given by

$$
\begin{aligned}
& \mathcal{O}_{i}(x) \rightarrow \mathcal{O}_{i}(x, N)=\phi_{x}^{\dagger}\left(\prod_{n=0}^{N-1} U_{x+n i, i}\right) \phi_{x+N i}, \\
& \overline{\mathcal{O}}_{i}(x) \rightarrow \overline{\mathcal{O}}_{i}(x, N)=\phi_{x}^{\dagger}\left(\prod_{n=1}^{N} U_{x-n i, i}^{\dagger}\right) \phi_{x-N i},
\end{aligned}
$$

for which Eqs. (61) and (62) do not change (in the following, in order to simplify the notations, the dependence on $N$ is never displayed explicitly). Note that such highly nonlocal operators are seen to improve the signal significantly.

\section{OPERATORS}

A generic operator $\mathcal{O}_{\alpha}^{\Gamma}(x)$, transforming under a specific irrep $\Gamma$ of the group $\mathcal{G}$, obeys the following transformation law

$$
\mathcal{O}_{\alpha}^{\Gamma}(x) \rightarrow R_{\beta \alpha}(\mathcal{S}) O_{\beta}^{\Gamma}\left(x^{\prime}\right) .
$$

The prescription for constructing such operators is well known (see, e.g., Refs. $[3,8]$ ). Consider first the case of one-particle operators $\mathcal{O}(\mathbf{x}, t)$, whose transformation properties (a scalar, vector, etc.) are defined. More specifically, let the action of the group element $\mathcal{S}$ on the field $\mathcal{O}(\mathbf{x}, t)$ be represented by a linear matrix $A\left(\mathcal{S}^{-1}\right)$ [unit matrix for scalars, $T_{i j}\left(\mathcal{S}^{-1}\right)$ for vectors, etc.]. Then, it is possible to project out the component, contributing to a given irrep $\Gamma$. In momentum space, the corresponding expression takes the form 


$$
\mathcal{O}_{\alpha}^{\Gamma}(\mathbf{p}, t)=\sum_{x} e^{i \mathbf{p x}} \sum_{\mathcal{S} \in \mathcal{G}}\left(R_{\alpha \beta}^{\Gamma}(\mathcal{S})\right)^{*}\left(A\left(\mathcal{S}^{-1}\right) \mathcal{O}\right)(\mathbf{x}, t),
$$

where the set of $R_{\alpha \beta}^{\Gamma}(\mathcal{S})$ forms the irrep $\Gamma$ of the group $\mathcal{G}$ with index $\beta$ fixed.

The two-particle operator with total momentum $\mathbf{p}$ and relative momentum $\mathbf{q}$ is given by

$$
\begin{aligned}
& \mathcal{O}_{\alpha}^{\Gamma}(\mathbf{p}, \mathbf{q}, t) \\
& \quad=\sum_{\mathbf{x}, \mathbf{y}}\left(\sum_{\mathcal{S} \in \mathcal{G}} e^{i \mathbf{p} \mathbf{x}+\tilde{\mathbf{q}}(\mathbf{y}-\mathbf{x})}\right)\left(R_{\alpha \beta}^{\Gamma}(\mathcal{S})\right)^{*}\left(A\left(\mathcal{S}^{-1}\right) \mathcal{O}\right)(\mathbf{x}, \mathbf{y}, t),
\end{aligned}
$$

where the vector $\tilde{\mathbf{q}}$ is obtained from the vector $\mathbf{q}$ via $\tilde{q}_{i}=T_{i j}\left(\mathcal{S}^{-1}\right) q_{j}$.

In order to simplify the construction of the operators, we note that the irreducible operators transform exactly as the basis vectors in the corresponding irrep. We shall illustrate the procedure with one example. Consider the construction of the two-particle operator in the case where the momenta of the particles are $\frac{2 \pi}{L}(0,0,1)$ and $\frac{2 \pi}{L}(0,0,0)$. This is a case of the little group $C_{4 v}$. From Table XIII one finds that, e.g., the state $|2,0\rangle$ is the basis vector in the irrep $A_{1}$. On the other hand, various linear combinations of the Cartesian components of the vector field $V_{i}(\mathbf{x}, t)$ transform as

$$
\begin{aligned}
& |1, \pm 1\rangle \sim \mp \frac{1}{\sqrt{2}}\left(V_{1}(\mathbf{x}, t) \pm i V_{2}(\mathbf{x}, t)\right), \\
& |1, \pm 0\rangle \sim V_{3}(\mathbf{x}, t) .
\end{aligned}
$$

The state $|2,0\rangle$ can be obtained as a linear combination of the spin-1 states:

$|2,0\rangle=\frac{1}{\sqrt{6}}|1,1\rangle|1,-1\rangle+\sqrt{\frac{2}{3}}|1,0\rangle|1,0\rangle+\frac{1}{\sqrt{6}}|1,-1\rangle|1,1\rangle$.

Taking into account the Eq. (67), we finally obtain that the following operator

TABLE III. Complete list of one-particle operators in the multiple moving frames and irreps.

\begin{tabular}{lcc}
\hline \hline $\mathbf{d}$ & $\Gamma$ & Operator \\
\hline$(0,0,0)$ & $T_{1}^{-}$ & $V_{i}(\mathbf{x}, t)$ \\
$(0,0,1)$ & $A_{1}$ & $V_{3}(\mathbf{x}, t)$ \\
& $E$ & $V_{1}(\mathbf{x}, t)+V_{2}(\mathbf{x}, t)$ \\
$(1,1,0)$ & $A_{1}(\mathbf{x}, t)-V_{2}(\mathbf{x}, t)$ \\
& $B_{1}$ & $V_{1}(\mathbf{x}, t)+V_{2}(\mathbf{x}, t)$ \\
& $B_{2}$ & $V_{1}(\mathbf{x}, t)-V_{2}(\mathbf{x}, t)$ \\
$(1,1,1)$ & $A_{1}$ & $V_{3}(\mathbf{x}, t)$ \\
& $E$ & $\sum_{i=1}^{3} V_{i}(\mathbf{x}, t)$ \\
& & $V_{1}(\mathbf{x}, t)-V_{2}(\mathbf{x}, t)$ \\
\hline \hline
\end{tabular}

$$
\begin{aligned}
\mathcal{O}^{A_{1}}(\mathbf{p}, t)= & \sum_{\mathbf{x}, \mathbf{y}} e^{i \mathbf{p x}}\left(-V_{1}(\mathbf{x}, t) V_{1}(\mathbf{y}, t)\right. \\
& \left.-V_{2}(\mathbf{x}, t) V_{2}(\mathbf{y}, t)+2 V_{3}(\mathbf{x}, t) V_{3}(\mathbf{y}, t)\right),
\end{aligned}
$$

with $\mathbf{p}=\frac{2 \pi}{L}(0,0,1)$, indeed projects on the irrep $A_{1}$ of the group $C_{4 v}$.

We have collected one- and two-particle operators in Tables III and V, respectively. Note that this simplified procedure is only possible if one of the momenta is zero or both are in the same little group; if that is not the case, one must use Eq. (66), as in the case for $\Gamma=A_{1}, \mathbf{p}=\frac{2 \pi}{L}(1,1,0)$ and $\mathbf{q}=\frac{2 \pi}{L}(1,1,0)$ of Table V.

\section{NUMERICAL RESULTS}

The parameter sets we use are compiled in Table IV. Note that we have five different sets of bare parameters $\lambda$ and $\kappa$ for $L=12$ and $T=24$. For one of these parameter sets we have a second volume with $L=16$ and $T=32$. We compute correlation functions

$$
C^{\Gamma}\left(t-t^{\prime}\right)=\left\langle\mathcal{O}^{\Gamma}(t)\left(\mathcal{O}^{\Gamma}\right)^{\dagger}\left(t^{\prime}\right)\right\rangle
$$

using the operators defined in the previous sections. At large time differences $t-t^{\prime}$ these correlation functions are proportional to $\exp \left(-E\left(t-t^{\prime}\right)\right)$ with $E$ the energy of the lowest state with the corresponding quantum numbers. The energies are calculated with a fit to the shifted correlation function

$$
\tilde{C}^{\Gamma}(t)=C^{\Gamma}(t)-C^{\Gamma}(t+1),
$$

including an excited state, and the errors are calculated using the Jackknife method. We use the shifted correlation function to subtract any contribution constant in time stemming from vacuum expectation values; see also Ref. [24]. Thermal contaminations in the two-particle correlation functions with nonzero total momentum turn out to be not important for our analysis (see plots in Appendix F). Therefore, we have used the shifted correlation function throughout. All the results are listed in the tables of Appendix D and they will be discussed in this section. In addition, we show exemplary plots for effective masses for selected correlation functions in Appendix F. The effective mass $m_{\text {eff }}$ is calculated by solving

TABLE IV. Ensembles used for the simulations. The gauge coupling is kept constant, $\beta=2.5$.

\begin{tabular}{llrllll}
\hline \hline$L^{3} \times T$ & Reference & \multicolumn{1}{c}{$\mathrm{N}$} & $m_{0}^{2}$ & \multicolumn{1}{c}{$\lambda_{c}$} & $\kappa$ & $\lambda$ \\
\hline $16^{3} \times 32$ & A16 & 138000 & -35 & 88 & 0.18425 & 2.9873 \\
& A12 & 33000 & -35 & 88 & 0.18425 & 2.9873 \\
& B12 & 24000 & -35.5 & 90.6 & 0.18208 & 3.0036 \\
$12^{3} \times 24$ & C12 & 21500 & -35.6 & 91.6 & 0.18084 & 2.9956 \\
& D12 & 16000 & -35.85 & 93.1 & 0.17949 & 2.9994 \\
& E12 & 18800 & -36.1 & 94.7 & 0.17802 & 3.0012 \\
\hline \hline
\end{tabular}


TABLE V. List of used two-particle operators in the multiple moving frames and irreducible representations. The operator $\mathcal{O}^{\Gamma}(\mathbf{p}, \mathbf{q}, t)$ is built from the position-space operators, given in this table, by calculating the Fourier-transform with $e^{i \mathbf{p x}+i \mathbf{q}(\mathbf{y}-\mathbf{x})}$. This prescription holds for all operators except with $\mathbf{p}=\frac{2 \pi}{L}(1,1,0)$ and $\mathbf{q}=\frac{2 \pi}{L}(0,1,0)$ (the second line from below), for which the shortcut is no more applicable and one has to use Eq. (66).

\begin{tabular}{|c|c|c|c|c|}
\hline d & $\Gamma$ & $\frac{L}{2 \pi} \mathbf{p}$ & $\frac{L}{2 \pi} \mathbf{q}$ & $\mathcal{O}^{\Gamma}(\mathbf{x}, \mathbf{y}, t)$ \\
\hline$(0,0,0)$ & $\begin{array}{l}A_{1} \\
E^{+} \\
T_{2}^{+}\end{array}$ & $\begin{array}{l}\mathbf{0} \\
\mathbf{0} \\
\mathbf{0}\end{array}$ & $\begin{array}{l}\mathbf{0} \\
\mathbf{0} \\
\mathbf{0}\end{array}$ & $\begin{array}{c}\sum_{i=1}^{3} V_{i}(\mathbf{x}, t) V_{i}(\mathbf{y}, t) \\
V_{1}(\mathbf{x}, t) V_{1}(\mathbf{y}, t)-V_{2}(\mathbf{x}, t) V_{2}(\mathbf{y}, t) \\
V_{1}(\mathbf{x}, t) V_{2}(\mathbf{y}, t)+V_{2}(\mathbf{x}, t) V_{1}(\mathbf{y}, t)\end{array}$ \\
\hline$(0,0,1)$ & $\begin{array}{c}A_{1} \\
A_{1} \\
A_{2} \\
B_{1} \\
B_{2} \\
E\end{array}$ & $\begin{array}{l}(0,0, n) \\
(0,0, n) \\
(0,0, n) \\
(0,0, n) \\
(0,0, n) \\
(0,0, n)\end{array}$ & $\begin{array}{l}(0,0, m) \\
(0,0, m) \\
(0,0, m) \\
(0,0, m) \\
(0,0, m) \\
(0,0, m)\end{array}$ & $\begin{array}{c}\left.V_{1}(\mathbf{x}, t) V_{1}(\mathbf{y}, t)+V_{2}(\mathbf{x}, t) V_{2}(\mathbf{y}, t)+V_{3}(\mathbf{x}, t) V_{3}(\mathbf{y}, t)\right) \\
\left.-V_{1}(\mathbf{x}, t) V_{1}(\mathbf{y}, t)-V_{2}(\mathbf{x}, t) V_{2}(\mathbf{y}, t)+2 V_{3}(\mathbf{x}, t) V_{3}(\mathbf{y}, t)\right) \\
V_{1}(\mathbf{x}, t) V_{2}(\mathbf{y}, t)-V_{2}(\mathbf{x}, t) V_{1}(\mathbf{y}, t) \\
V_{1}(\mathbf{x}, t) V_{1}(\mathbf{y}, t)-V_{2}(\mathbf{x}, t) V_{2}(\mathbf{y}, t) \\
V_{1}(\mathbf{x}, t) V_{2}(\mathbf{y}, t)+V_{2}(\mathbf{x}, t) V_{1}(\mathbf{y}, t) \\
V_{3}(\mathbf{x}, t)\left(V_{1}(\mathbf{y}, t)+V_{2}(\mathbf{y}, t)\right)+\left(V_{1}(\mathbf{x}, t)+V_{2}(\mathbf{x}, t)\right) V_{3}(\mathbf{y}, t)\end{array}$ \\
\hline$(1,1,0)$ & $\begin{array}{l}A_{1} \\
A_{1} \\
A_{2}\end{array}$ & $\begin{array}{l}(1,1,0) \\
(1,1,0) \\
(1,1,0)\end{array}$ & $\begin{array}{c}\mathbf{0} \\
(0,1,0) \\
\mathbf{0}\end{array}$ & $\begin{array}{c}V_{3}(\mathbf{x}, t) V_{3}(\mathbf{y}, t) \\
V_{3}(\mathbf{x}, t)\left(V_{1}(\mathbf{y}, t)-V_{2}(\mathbf{y}, t)\right)+\left(V_{1}(\mathbf{x}, t)-V_{2}(\mathbf{x}, t)\right) V_{3}(\mathbf{y}, t)\end{array}$ \\
\hline
\end{tabular}

$\frac{C^{\Gamma}(t)-C^{\Gamma}(t+1)}{C^{\Gamma}(t+1)-C^{\Gamma}(t+2)}=\frac{\sinh \left(m_{\mathrm{eff}}(t-T / 2+a / 2)\right)}{\sinh \left(m_{\mathrm{eff}}(t+1-T / 2+a / 2)\right)}$

numerically for $m_{\mathrm{eff}}$.

\section{A. One-particle results}

In Fig. 1(a) we show the dependence of the mass of the vector particle $m_{v}$ on the length of the operator in Eq. (63) for ensemble A12. We observe a clear improvement of the signal with increasing operator length. When using moving frames, the best signal is empirically seen at $N=L /(d+1)$, with $d$ being the units of momentum in that particular direction.

Similarly, in Fig. 1(c) we show the dependence of the mass of the scalar particle $m_{s}$ on the length of the operator in Eq. (61), N, for ensemble A12. The same mass can be measured using operator $\mathcal{O}=|\phi|$. In addition we show the mass measured using the operator $\mathcal{O}^{A_{1}}(0,0, t)$. The corresponding results are shown in the same figure as horizontal lines denoted as $m(\phi)$ for the operator $\mathcal{O}=|\phi|$ and $m_{W}^{A_{1}}$ for operator $\mathcal{O}^{A_{1}}(0,0, t)$, respectively. For $m_{s}$ the signal improves again with increasing operator length. However, while $m_{s}$ agrees with $m(\phi)$ up to operator lengths of nine, at lengths larger than nine its values drops and finally agrees with $m_{W}^{A_{1}}$. It is expected that these two states mix because they have the same quantum numbers. Hence, it seems that the overlap of the operator Eq. (61) shifts with increasing operator length due to the presence of more gauge links. Moreover, since the mass of the scalar is only needed as a reference, and the gap between the scalar and the vector mass is big, we do not perform a variational analysis at this point.
In Fig. 1(b) we compare the masses of a single vector and scalar particle as a function of $\kappa$ for $L=12$. With increasing $\kappa$-value we observe the vector mass to be approximately constant while the scalar mass increases almost linearly. In the range of $\kappa$-values studied here the vector mass value is always smaller than the scalar mass value. We recall that in the continuum the bare masses of the particles are given by $m_{\phi}^{2}=-2 m_{0}^{2}$ and $m_{V}^{2}=-\frac{g^{2} m_{0}^{2}}{\lambda_{c}}$, respectively. Hence, we expect the mass of the vector to be suppressed with respect to the scalar mass by a factor $g$ and $1 / \lambda_{c}$. However, it is not clear why the scalar mass duplicates with increasing $\kappa$, whereas the vector mass increases at best slightly.

In Fig. 1(d) we show the energies of a single vector particle in the first moving frame as a function of $\kappa$ for $L=12$ for irreps $E$ and $A_{1}$. The (red) crosses represent the prediction by the continuum dispersion relation with the rest frame mass (irrep $T_{1}^{-}$) as input. For the moving frames we observe larger statistical uncertainties as compared to the dispersion relation. The energy splittings between different irreps and to the dispersion relation prediction are compatible with zero.

The comparison between different irreps is shown in more detail in Fig. 2(a): we plot $m_{v}$ for ensemble A16 for different irreps and center of mass momenta. Where we have several momenta for a given irrep, we also show the weighted average value. The values obtained for the different moving frames tend to be smaller than the one in the rest frame, although in every case but one they are compatible within $2 \sigma$. This discrepancy may be associated to discretization effects. In order to show this, in Fig. 2(b) we plot for ensemble A16 the energy of the vector particle in different irreps and moving frames together with the prediction from the continuum and lattice dispersion relations, the latter one reading 

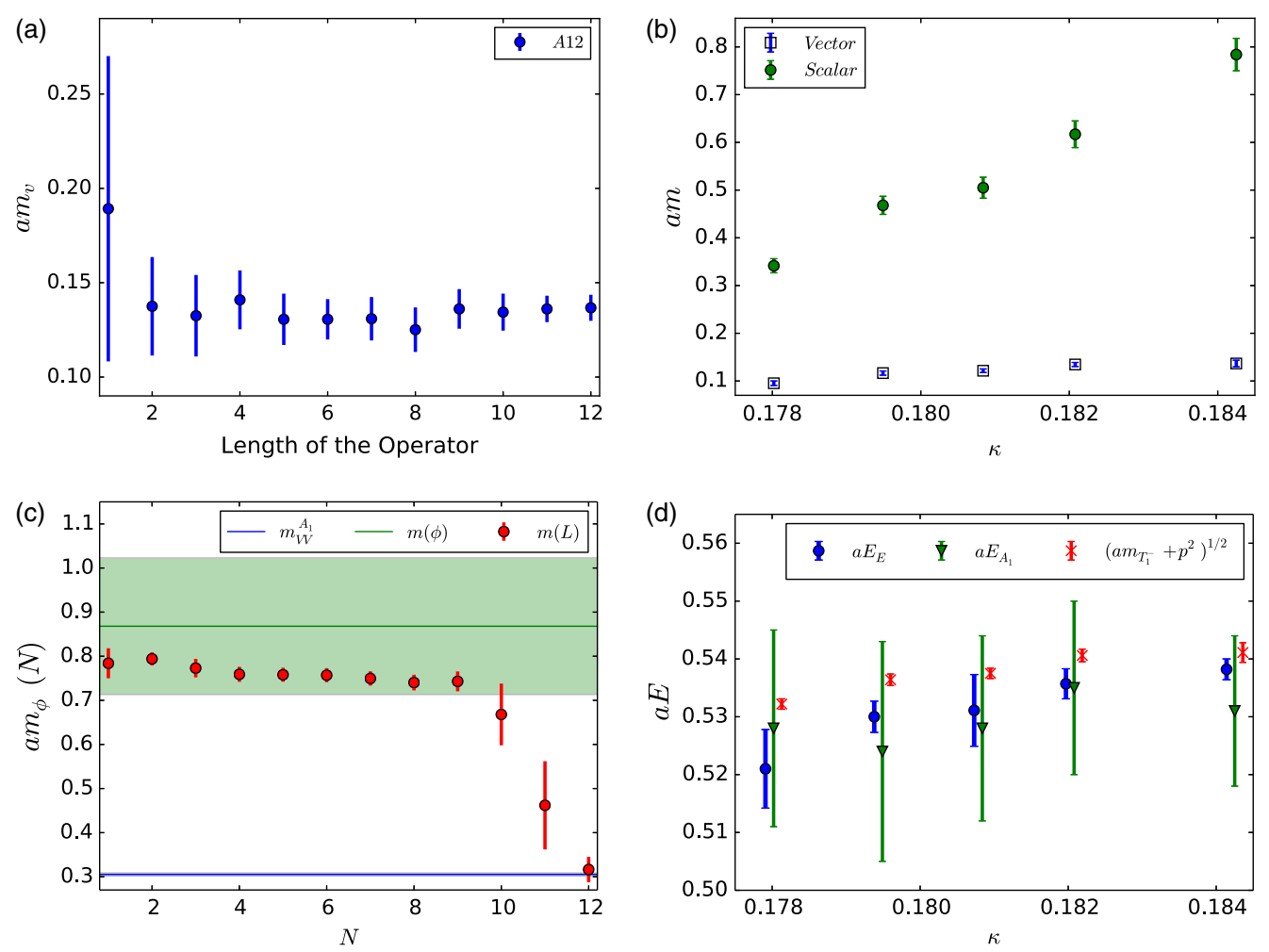

FIG. 1. Numerical results for single particle operators in ensembles with $L=12$. (a) Mass of the vector particle for ensemble A12 for different lengths of the operator in Eq. (62). (b) Mass of the scalar and vector particle for $L=12$ as a function of $\kappa$. (c) Mass of the scalar particle for ensemble A12 as a function of the length of the operator, $N$, as in Eq. (63). Further explanation can be found in the text. Note that the error in $m_{V V}^{A_{1}}$ is too small to be seen. (d) Energy of the vector particle for different irreps in the first moving frame for $L=12$ as a function of $\kappa$. It is also compared with the rest frame result by means of the continuum dispersion relation.

$$
\cosh a E=\cosh a m+(1-\cos a p) .
$$

Here, the mass am is taken to be the one measured in the rest frame, $m_{V}^{T_{1}^{+}}$. This shows that the continuum dispersion relation describes our data better and we do not observe large discretization effects for $a p<0.6$. However, around $a p \simeq 1$, this description becomes worse, as cut-off effects

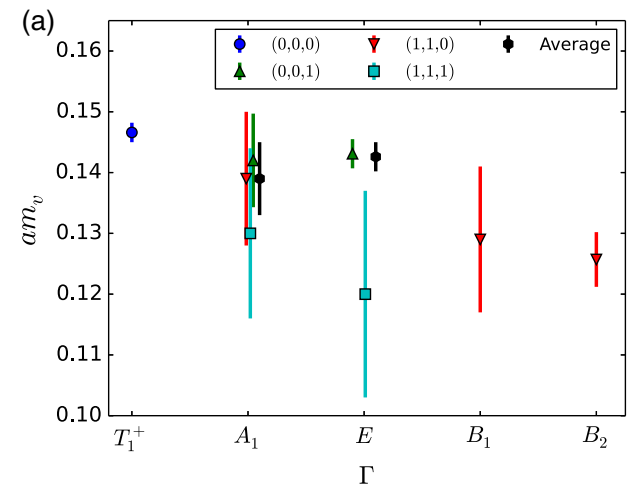

get bigger. From now on, we will always use the continuum dispersion relation with the mass as obtained from the rest frame as input.

\section{B. Two-particle results}

The energy difference $\Delta E$ is defined as the difference between the two-particle energy on the lattice and the

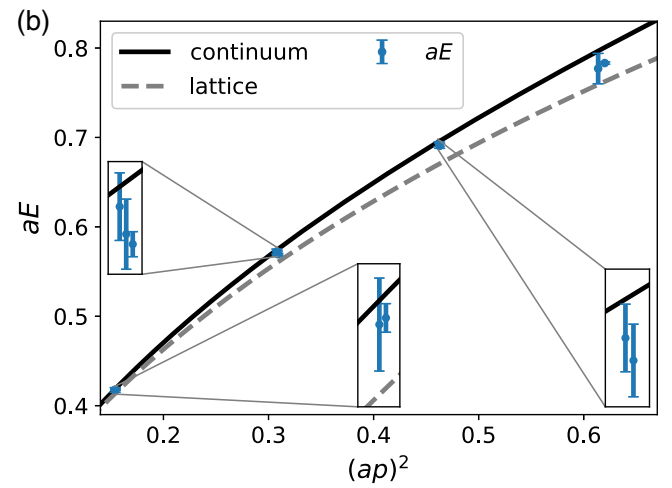

FIG. 2. Numerical results for single particle operators in ensemble A16. (a) Mass of the vector particle for different irreps and total momenta for ensemble A16. The continuum dispersion relation has been used. (b) Energies of a single vector particle as a function of the squared momentum $(a p)^{2}$. The solid and dashed lines represent the prediction of the continuum and lattice dispersion relations, respectively. Different irreps are slightly displaced for better readability. 
two-particle energy in absence of interactions. In Fig. 3(a) we show the results for $\Delta E$ as a function of $\kappa$ for the irreps $A_{1}, T_{2}^{+}$and $E^{+}$. For the largest $\kappa$-value we include both, A12 and A16 in the plot, respectively. For higher $\kappa$, the interaction leads to the positive shift $(\Delta E>0)$ with $\gtrsim 2 \sigma$ statistical significance. As $\kappa$ becomes smaller, all particles become lighter and the interaction seems to flip signs. For the lowest values of $\kappa$, the two-particle states have $\Delta E<0$.
Unfortunately, in the transition region $\Delta E$ is compatible with zero. Comparing A12 and A16, we see the expected volume dependence in the energy shift $\left(\Delta E \propto L^{-3}\right)$, when comparing $L=12$ with $L=16$. This can be inferred from Fig. 3(b), where we show $\Delta E$ for A12 and A16 as a function of $1 / L^{3}$.

In Fig. 3(e) we show $\Delta E$ for ensemble A16 in the first moving frame for the irreps $A_{1}, A_{2}, B_{1}, B_{2}$ and $E$. As
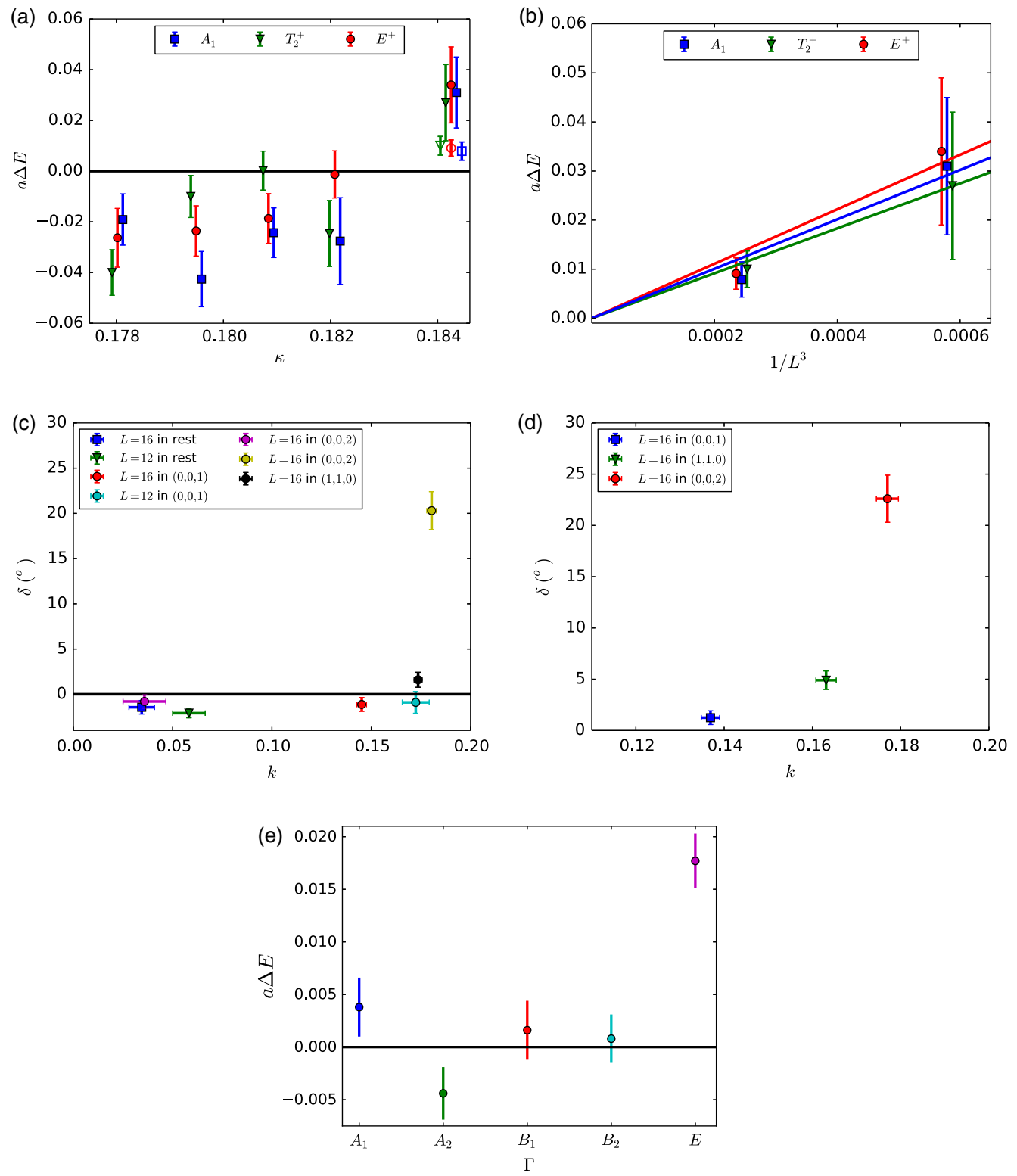

FIG. 3. Numerical results for two particles. (a) Energy difference $\Delta E$ as a function of $\kappa$ in the rest frame for different irreps. Open symbols correspond to ensemble A16 and closed ones to $L=12$. (b) Energy difference $\Delta E$ for ensemble A16 and A12 as a function of the length of the box, $L$. Additionally, we include the expected behavior. (c) Phase shift in the $J^{P}=0^{+}$channel in ensembles A12, A16 as a function of the scattering momentum $k$. Partial waves $J>1$ have been neglected and the two possible $L, S$ combinations cannot be distinguished. (d) Phase shift in the $J^{P}=0^{-}$channel in ensemble A16 as a function of the scattering momentum $k$. They are calculated neglecting partial waves $J>1$. (e) Energy difference $\Delta E$ for ensemble A16 in the moving frame with total momentum $p=\frac{2 \pi}{L}(0,0,1)$ and $q=0$ as in Eq. (66) for different irreps $\Gamma$. 
expected, $\Delta E$ depends on the considered irrep. For the moving frame shown in Fig. 3(e) $\Delta E$ is only significantly different from zero for the $E$ irrep.

Subsequently, in Fig. 3(c) we show the phase shifts with $J^{P}=0^{+}$computed from the energy shifts. Note that we neglect partial waves with $J>1$ and that the two possible $L, S$ combinations cannot be distinguished at this level:

$$
\cot \delta_{0^{+}}=\omega_{00} .
$$

For the highest momentum shown, the ratio between the nonrelativistic kinetic energy and the mass is quite large, $\frac{E_{k}}{m} \approx 0.8$. Hence, the kinematic suppression of higher partial waves, though present, is not strong anymore and a corresponding systematical error is to be expected. The phase shift appears to be small and negative for small scattering momentum, indicating a weak repulsive interaction. If the data for large $k$ can be taken seriously, there seems to be a flip of sign around scattering momentum $a k \approx 0.18$ and a rapid growth toward pi/2 beyond this point. Without further study, we cannot say whether this corresponds to a resonance or not.

Finally, in Fig. 3(d) we show the results for the phase shift with $J^{P}=0^{-}$. In this channel there are no mixings, and one can expect a cleaner determination with respect to $J^{P}=0^{+}$. Again, we neglect partial waves with $J>1$ :

$$
\cot \delta_{0^{-}}=\omega_{00} .
$$

The phase shift values are consistent with an attractive interaction. $\delta$ increases with increasing $k$, which might indicate a resonance for $a k>0.18$.

\section{SUMMARY AND OUTLOOK}

In this paper we have rederived the Lüscher formalism for particles with general spin. We find complete agreement with Ref. [9]. We have explicitly formulated this approach for the case of two vector particles in the scalar channel.

The formalism is applied to scalar QED in the Higgs phase, where the gauge boson becomes massive. For this model we derived the relevant operators to study scalar, vector and two vector particles with center of mass momenta up to $a \mathbf{p}=\frac{2 \pi}{L}(1,1,1)$. We have simulated scalar QED using Markov chains and we have estimated interacting and noninteracting energy levels for various total momenta in the scalar channel. We have studied a set of bare parameter values and two volumes. Even though the model is sufficiently simple to simulate, it is still a challenge to gather enough statistics to obtain significant results. In general, the correlation functions measured by us appear to be rather noisy.

In addition to the noise, it turns out that we are facing a dependence of the estimated single particle energy levels on the total momentum, which could be explained with lattice artifacts. In the energy shift $\Delta E$, this dependence is much less pronounced. However, statistical uncertainties are also larger for $\Delta E$. Still, $\Delta E$ shows the expected dependence on $L$. This makes us confident that our measurements are meaningful to a certain extent. Neglecting at this level any mixings and higher partial waves, we could extract the phase shift as a function of the scattering momentum.

A model-independent determination of all $S$-matrix parameters is computationally very expensive. Namely, it would require many volumes and the use of the multichannel effective-range expansion [Eq. (29)]. However, our results show that it is feasible to study the interaction of two vector particles. Hence, in the future, we plan to apply these ideas to study the possibility of the Higgs boson to be a bound state of two $W$ bosons.

\section{ACKNOWLEDGMENTS}

We would like to acknowledge the lattice group in Bonn and Roberto Frezzotti for the interesting discussions and the support provided. This work was supported in part by the DFG in the Sino-German CRC110, by Volkswagenstiftung under Contract No. 93562 and by Shota Rustaveli National Science Foundation (SRNSF), Grant No. DI-2016-26. Part of the computer time for this project was made available to us by Jureca in Jülich. Finally, special thanks to BCGS for the continuous support.

\section{APPENDIX A: CONVENTION FOR THE IRREDUCIBLE REPRESENTATIONS}

The ten irreducible representations (irreps) of the spatial symmetry groups of the lattice include 4 one-dimensional, 2 two-dimensional and 4 three-dimensional ones. They are:

(i) $A_{1}$ is the trivial representation, where all elements of $O_{h}$ are 1 .

(ii) $A_{2}$ is the trivial representation for $O$ times -1 when an inversion is present.

(iii) $B_{1}$ assigns $R_{i}=-1$ to rotations in the conjugacy classes $6 C_{4}$ and $6 C_{2}^{\prime}$ and $R=1$ otherwise.

(iv) $B_{2}$ is the same as $B_{1}$ multiplying by -1 when an inversion is present.

(v) $E$ labels a two-dimensional representation. For the octahedral group, the superscript $E^{ \pm}$means whether an inversion multiplies the element by \pm 1 .

(vi) $T_{1}^{ \pm}$is a three-dimensional representation which coincides with the Wigner matrices: $R_{i}=\exp \left(-i n^{i} J \omega_{i}\right)$, with $J$ the group generators and $n^{i}$ and $\omega_{i}$ as listed in Table VI. The superscript \pm labels whether spatial inversion are assigned always +1 or \pm 1 .

(vii) $T_{2}^{ \pm}$is the same as $T_{1}$ with a change of sign in the conjugacy classes $6 C_{4}$ and $6 C_{2}^{\prime}$.

In Appendix B the elements and characters of the different spatial symmetry groups are shown. They are taken to be in agreement with Ref. $[3,8]$. 


\section{APPENDIX B: GROUP TABLES}

TABLE VI. Rotations of the group $O$. Last column includes the element of the two dimensional irreducible representation for the cubic group.

\begin{tabular}{|c|c|c|c|c|}
\hline Class & $R_{i}$ & $n$ & $\omega$ & $R_{i}\left(E^{ \pm}\right)$ \\
\hline I & 1 & any & 0 & 1 \\
\hline \multirow[t]{8}{*}{$8 C_{3}$} & 2 & $(1,1,1)$ & $-2 \pi / 3$ & $-\frac{1}{2} \mathbf{1}+\frac{i \sqrt{3}}{2} \sigma_{2}$ \\
\hline & 3 & $(1,1,1)$ & $2 \pi / 3$ & $-\frac{1}{2} \mathbf{1}-\frac{i \sqrt{3}}{2} \sigma_{2}$ \\
\hline & 4 & $(-1,1,1)$ & $-2 \pi / 3$ & $-\frac{1}{2} \mathbf{1}-\frac{i \sqrt{3}}{2} \sigma_{2}$ \\
\hline & 5 & $(-1,1,1)$ & $2 \pi / 3$ & $-\frac{1}{2} \mathbf{1}+\frac{i \sqrt{3}}{2} \sigma_{2}$ \\
\hline & 6 & $(-1,-1,1)$ & $-2 \pi / 3$ & $-\frac{1}{2} \mathbf{1}+\frac{i \sqrt{3}}{2} \sigma_{2}$ \\
\hline & 7 & $(-1,-1,1)$ & $2 \pi / 3$ & $-\frac{1}{2} \mathbf{1}-\frac{i \sqrt{3}}{2} \sigma_{2}$ \\
\hline & 8 & $(1,-1,1)$ & $-2 \pi / 3$ & $-\frac{1}{2} \mathbf{1}-\frac{i \sqrt{3}}{2} \sigma_{2}$ \\
\hline & 9 & $(1,-1,1)$ & $2 \pi / 3$ & $-\frac{1}{2} \mathbf{1}+\frac{i \sqrt{3}}{2} \sigma_{2}$ \\
\hline \multirow[t]{6}{*}{$6 C_{4}$} & 10 & $(1,0,0)$ & $-\pi / 2$ & \\
\hline & 11 & $(1,0,0)$ & $\pi / 2$ & $-\frac{1}{2} \sigma_{3}-\frac{\sqrt{3}}{2} \sigma_{1}$ \\
\hline & 12 & $(0,1,0)$ & $-\pi / 2$ & $-\frac{1}{2} \sigma_{3}+\frac{\sqrt{3}}{2} \sigma_{1}$ \\
\hline & 13 & $(0,1,0)$ & $\pi / 2$ & $-\frac{1}{2} \sigma_{3}+\frac{\sqrt{3}}{2} \sigma_{1}$ \\
\hline & 14 & $(0,0,1)$ & $-\pi / 2$ & $\sigma_{3}$ \\
\hline & 15 & $(0,0,1)$ & $\pi / 2$ & $\sigma_{3}$ \\
\hline \multirow[t]{6}{*}{$6 C_{2}^{\prime}$} & 16 & $(0,1,1)$ & $-\pi$ & $-\frac{1}{2} \sigma_{3}-\frac{\sqrt{3}}{2} \sigma_{1}$ \\
\hline & 17 & $(0,-1,1)$ & $-\pi$ & $-\frac{1}{2} \sigma_{3}-\frac{\sqrt{3}}{2} \sigma_{1}$ \\
\hline & 18 & $(1,1,0)$ & $-\pi$ & $\sigma_{3}$ \\
\hline & 19 & $(1,-1,0)$ & $-\pi$ & $\sigma_{3}$ \\
\hline & 20 & $(1,0,1)$ & $-\pi$ & $-\frac{1}{2} \sigma_{3}+\frac{\sqrt{3}}{2} \sigma_{1}$ \\
\hline & 21 & $(-1,0,1)$ & $-\pi$ & $-\frac{1}{2} \sigma_{3}+\frac{\sqrt{3}}{2} \sigma_{1}$ \\
\hline \multirow[t]{3}{*}{$3 C_{2}$} & 22 & $(1,0,0)$ & $-\pi$ & 1 \\
\hline & 23 & $(0,1,0)$ & $-\pi$ & 1 \\
\hline & 24 & $(0,0,1)$ & $-\pi$ & 1 \\
\hline
\end{tabular}

TABLE VII. Elements of the little groups.

\begin{tabular}{lccc}
\hline \hline Group & Boost & Name & Elements \\
\hline$O_{h}$ & $(0,0,1)$ & $C_{4 v}$ & $R_{1}, R_{14}, R_{15}, R_{24}, I R_{18}, I R_{19}, I R_{22}, I R_{23}$ \\
& $(1,1,0)$ & $C_{2 v}$ & $R_{1}, R_{18}, I R_{19}, I R_{24}$ \\
& $(1,1,1)$ & $C_{3 v}$ & $R_{1}, R_{2}, R_{3}, I R_{17}, I R_{19}, I R_{21}$ \\
\hline \hline
\end{tabular}

TABLE VIII. Elements of the two dimensional irrep in the little groups.

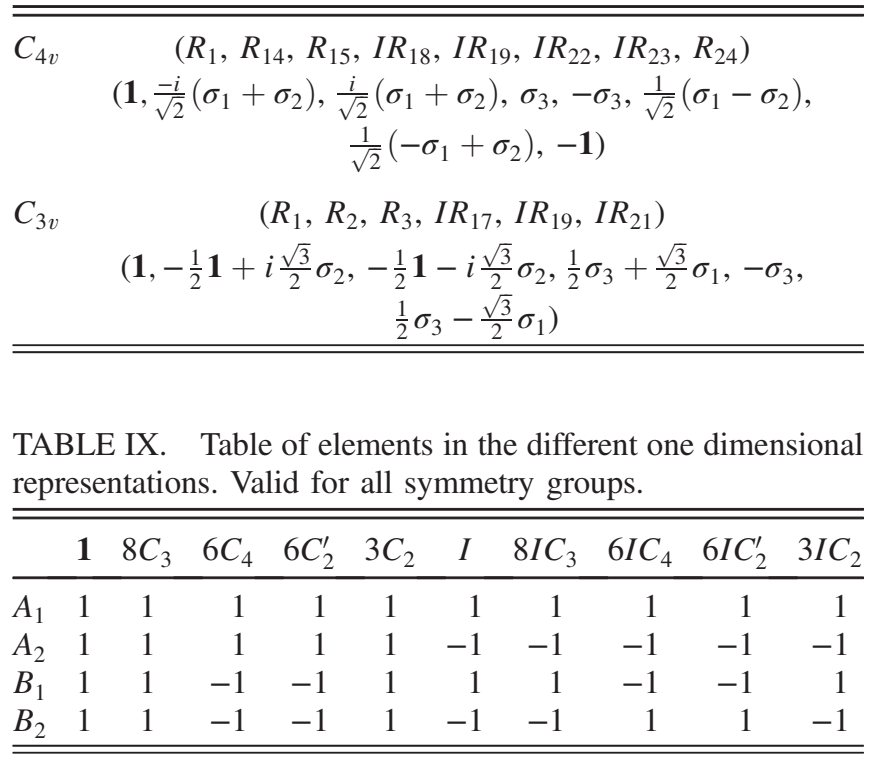

\section{APPENDIX C: BASIS VECTORS}

TABLE X. Basis vectors for $O_{h}$ with $(-1)^{J} \neq(-1)^{l}$.

\begin{tabular}{llc}
\hline \hline$\Gamma$ & $J$ & Basis vectors \\
\hline$A_{2}$ & 0 & $|0,0\rangle$ \\
$T_{1}^{+}$ & 1 & $-\frac{1}{\sqrt{2}}(|1,1\rangle-|1,-1\rangle)$ \\
& 1 & $|1,0\rangle$ \\
& 1 & $\frac{i}{\sqrt{2}}(|1,1\rangle+|1,-1\rangle)$ \\
$T_{2}^{-}$ & 2 & $\frac{1}{\sqrt{2}}(|2,1\rangle+|2,-1\rangle)$ \\
& 2 & $\frac{i}{\sqrt{2}}(|2,1\rangle-|2,-1\rangle)$ \\
& 2 & $\frac{1}{\sqrt{2}}(|2,-2\rangle-|2,2\rangle)$ \\
$E^{-}$ & 2 & $\frac{1}{\sqrt{2}}(|2,-2\rangle+|2,2\rangle)$ \\
& 2 & $|2,0\rangle$ \\
\hline \hline
\end{tabular}

TABLE XI. Basis vectors for $O_{h}$ with $(-1)^{J}=(-1)^{l}$.

\begin{tabular}{llc}
\hline \hline$\Gamma$ & $J$ & Basis vectors \\
\hline$A_{1}$ & 0 & $|0,0\rangle$ \\
$T_{1}^{-}$ & 1 & $-\frac{1}{\sqrt{2}}(|1,1\rangle-|1,-1\rangle)$ \\
& 1 & $|1,0\rangle$ \\
& 1 & $\frac{i}{\sqrt{2}}(|1,1\rangle+|1,-1\rangle)$ \\
$T_{2}^{+}$ & 2 & $\frac{1}{\sqrt{2}}(|2,1\rangle+|2,-1\rangle)$ \\
& 2 & $\frac{i}{\sqrt{2}}(|2,1\rangle-|2,-1\rangle)$ \\
& 2 & $\frac{1}{\sqrt{2}}(|2,-2\rangle-|2,2\rangle)$ \\
$E^{+}$ & 2 & $\frac{1}{\sqrt{2}}(|2,-2\rangle+|2,2\rangle)$ \\
& 2 & $|2,0\rangle$ \\
\hline \hline
\end{tabular}


TABLE XII. Basis vectors for $C_{4 v}$ with $(-1)^{J} \neq(-1)^{l}$.

\begin{tabular}{llcc}
\hline \hline$\Gamma$ & $J$ & $\alpha$ & Basis vectors \\
\hline$A_{2}$ & 0 & & $|0,0\rangle$ \\
$A_{2}$ & 1 & & $|1,0\rangle$ \\
$E$ & 1 & 1 & $\frac{1}{2}(1+i)|1,-1\rangle-\frac{1}{2}(1-i)|1,1\rangle$ \\
& 1 & 2 & $\frac{1}{\sqrt{2}}|1,-1\rangle-\frac{1}{\sqrt{2}} i|1,1\rangle$ \\
$A_{2}$ & 2 & & $|2,0\rangle$ \\
$B_{1}$ & 2 & & $\frac{1}{\sqrt{2}}(|2,-2\rangle-|2,2\rangle)$ \\
$B_{2}$ & 2 & & $\frac{1}{\sqrt{2}}(|2,-2\rangle+|2,2\rangle)$ \\
$E$ & 2 & 1 & $\frac{1}{2}(1-i)|2,-1\rangle-\frac{1}{2}(1+i)|2,1\rangle$ \\
& 2 & 2 & $\frac{1}{\sqrt{2}}|2,-1\rangle+\frac{i}{\sqrt{2}}|2,1\rangle$ \\
\hline \hline
\end{tabular}

TABLE XV. Basis vectors for $C_{2 v}$ with $(-1)^{J}=(-1)^{l}$.

\begin{tabular}{llcc}
\hline \hline$\Gamma$ & $J$ & $n_{\Gamma}$ & Basis vectors \\
\hline$A_{1}$ & 0 & & $|0,0\rangle$ \\
$A_{1}$ & 1 & $\frac{1}{\sqrt{2}}|1,-1\rangle+\frac{i}{\sqrt{2}}|1,1\rangle$ \\
$B_{1}$ & 1 & & $\frac{1}{\sqrt{2}}|1,-1\rangle-\frac{i}{\sqrt{2}}|1,1\rangle$ \\
$B_{2}$ & 1 & & $|1,0\rangle$ \\
$A_{1}$ & 2 & 1 & $|2,0\rangle$ \\
$A_{1}$ & 2 & 2 & $\frac{1}{\sqrt{2}}(|2,-2\rangle-|2,2\rangle)$ \\
$A_{2}$ & 2 & & $\frac{1}{\sqrt{2}}(|2,-1\rangle-i|2,1\rangle)$ \\
$B_{1}$ & 2 & & $\frac{1}{\sqrt{2}}(|2,-2\rangle+|2,2\rangle)$ \\
$B_{2}$ & 2 & & $\frac{1}{\sqrt{2}}(|2,-1\rangle+i|2,1\rangle)$ \\
\hline \hline
\end{tabular}

TABLE XIII. Basis vectors for $C_{4 v}$ with $(-1)^{J}=(-1)^{l}$.

\begin{tabular}{llcc}
\hline \hline$\Gamma$ & $J$ & $\alpha$ & Basis vectors \\
\hline$A_{1}$ & 0 & & $|0,0\rangle$ \\
$A_{1}$ & 1 & & $|1,0\rangle$ \\
$E$ & 1 & 1 & $\frac{1}{2}(1+i)|1,-1\rangle+\frac{1}{2}(1-i)|1,1\rangle$ \\
& 1 & 2 & $\frac{1}{\sqrt{2}}|1,-1\rangle+\frac{1}{\sqrt{2}} i|1,1\rangle$ \\
$A_{1}$ & 2 & & $|2,0\rangle$ \\
$B_{1}$ & 2 & & $\frac{1}{\sqrt{2}}(|2,-2\rangle+|2,2\rangle)$ \\
$B_{2}$ & 2 & & $\frac{1}{\sqrt{2}}(|2,-2\rangle-|2,2\rangle)$ \\
$E$ & 2 & 1 & $\frac{1}{2}(1-i)|2,-1\rangle+\frac{1}{2}(1+i)|2,1\rangle$ \\
& 2 & 2 & $\frac{1}{\sqrt{2}}|2,-1\rangle-\frac{i}{\sqrt{2}}|2,1\rangle$ \\
\hline \hline
\end{tabular}

TABLE XVI. Basis vectors for $C_{3 v}$ with $(-1)^{J} \neq(-1)^{l}$.

\begin{tabular}{llc}
\hline \hline$\Gamma$ & $J$ & Basis vectors \\
\hline$A_{2}$ & 0 & $|0,0\rangle$ \\
$A_{2}$ & 1 & $\frac{1}{\sqrt{3}}|1,-1\rangle+\frac{1-i}{\sqrt{6}}|1,0\rangle+\frac{i}{\sqrt{3}}|1,1\rangle$ \\
$E$ & 1 & $\frac{-i}{\sqrt{6}}|1,-1\rangle+\frac{1+i}{\sqrt{3}}|1,0\rangle+\frac{1}{\sqrt{6}}|1,1\rangle$ \\
& 1 & $\frac{1}{\sqrt{2}}|1,-1\rangle-\frac{i}{\sqrt{2}}|1,1\rangle$ \\
$A_{2}$ & 2 & $\frac{1}{\sqrt{6}}|2,-2\rangle+\frac{1-i}{\sqrt{6}}|2,-1\rangle+\frac{1+i}{\sqrt{6}}|2,1\rangle-\frac{1}{\sqrt{6}}|2,2\rangle$ \\
$E$ & 2 & $|2,0\rangle$ \\
& 2 & $\frac{1}{\sqrt{2}}(|2,-2\rangle+|2,2\rangle)$ \\
$E$ & 2 & $\frac{1-i}{\sqrt{6}}|2,-2\rangle+\frac{i}{\sqrt{6}}|2,-1\rangle-\frac{1}{\sqrt{6}}|2,1\rangle+\frac{-1+i}{\sqrt{6}}|2,2\rangle$ \\
& 2 & $\frac{1}{\sqrt{2}}(|2,-1\rangle-i|2,1\rangle)$ \\
\hline \hline
\end{tabular}

TABLE XIV. Basis vectors for $C_{2 v}$ with $(-1)^{J} \neq(-1)^{l}$.

\begin{tabular}{llcc}
\hline \hline$\Gamma$ & $J$ & $n_{\Gamma}$ & Basis vectors \\
\hline$A_{2}$ & 0 & & $|0,0\rangle$ \\
$A_{2}$ & 1 & $\frac{1}{\sqrt{2}}|1,-1\rangle+\frac{i}{\sqrt{2}}|1,1\rangle$ \\
$B_{2}$ & 1 & & $\frac{1}{\sqrt{2}}|1,-1\rangle-\frac{i}{\sqrt{2}}|1,1\rangle$ \\
$B_{1}$ & 1 & & $|1,0\rangle$ \\
$A_{2}$ & 2 & 1 & $\frac{1}{\sqrt{2}}(|2,-2\rangle-|2,2\rangle)$ \\
$A_{2}$ & 2 & 2 & $\frac{1}{\sqrt{2}}(|2,-1\rangle-i|2,1\rangle)$ \\
$A_{1}$ & 2 & & $\frac{1}{\sqrt{2}}(|2,-2\rangle+|2,2\rangle)$ \\
$B_{2}$ & 2 & & $\frac{1}{\sqrt{2}}(|2,-1\rangle+i|2,1\rangle)$ \\
$B_{1}$ & 2 & & \\
\hline \hline
\end{tabular}

TABLE XVII. Basis vectors for $C_{3 v}$ with $(-1)^{J}=(-1)^{l}$.

\begin{tabular}{llc}
\hline \hline$\Gamma$ & $J$ & Basis vectors \\
\hline$A_{1}$ & 0 & $|0,0\rangle$ \\
$A_{1}$ & 1 & $\frac{1}{\sqrt{3}}|1,-1\rangle+\frac{1-i}{\sqrt{6}}|1,0\rangle+\frac{i}{\sqrt{3}}|1,1\rangle$ \\
$E$ & 1 & $\frac{-i}{\sqrt{2}}|1,-1\rangle-\frac{1}{\sqrt{2}}|1,1\rangle$ \\
& 1 & $\frac{1}{\sqrt{6}}|1,-1\rangle+\frac{-1+i}{\sqrt{3}}|1,0\rangle+\frac{i}{\sqrt{6}}|1,1\rangle$ \\
$A_{1}$ & 2 & $\frac{1}{\sqrt{6}}|2,-2\rangle+\frac{1-i}{\sqrt{6}}|2,-1\rangle+\frac{1+i}{\sqrt{6}}|2,1\rangle-\frac{1}{\sqrt{6}}|2,2\rangle$ \\
$E$ & 2 & $\frac{1}{\sqrt{2}}(|2,-1\rangle-i|2,1\rangle)$ \\
& 2 & $-\frac{1-i}{\sqrt{6}}|2,-2\rangle-\frac{i}{\sqrt{6}}|2,-1\rangle+\frac{1}{\sqrt{6}}|2,1\rangle+\frac{1-i}{\sqrt{6}}|2,2\rangle$ \\
$E$ & 2 & $\frac{-1}{\sqrt{2}}(|2,-2\rangle+|2,2\rangle)$ \\
& 2 & $|2,0\rangle$ \\
\hline \hline
\end{tabular}




\section{APPENDIX D: TABLES FOR THE NUMERICAL RESULTS}

TABLE XVIII. Mass of the scalar and vector particle for the different ensembles.

\begin{tabular}{lcr}
\hline \hline Ensemble & $a m_{V}$ & $a m_{\phi}$ \\
\hline A12 & $0.1367(69)$ & $0.784(34)$ \\
B12 & $0.1346(43)$ & $0.617(28)$ \\
C12 & $0.1215(38)$ & $0.505(22)$ \\
D12 & $0.1166(44)$ & $0.468(19)$ \\
E12 & $0.0953(48)$ & $0.342(15)$ \\
A16 & $0.1466(16)$ & $0.728(13)$ \\
\hline \hline
\end{tabular}

TABLE XIX. Energy in the $A_{1} / E$ irreps of $d=(0,0,1)$ for the ensembles of Table V.

\begin{tabular}{lcr}
\hline \hline Ensemble & $a E_{A_{1}}$ & $a E_{E}$ \\
\hline A12 & $0.531(13)$ & $0.5382(18)$ \\
B12 & $0.535(15)$ & $0.5357(26)$ \\
C12 & $0.528(16)$ & $0.5225(26)$ \\
D12 & $0.524(19)$ & $0.5300(27)$ \\
E12 & $0.528(17)$ & $0.5210(68)$ \\
\hline \hline
\end{tabular}

TABLE XX. Energy and energy shift for two particles.

\begin{tabular}{llccrrr}
\hline \hline & \multicolumn{1}{c}{$a E_{A_{1}}$} & $a \Delta E_{A_{1}}$ & $a E_{E^{+}}$ & $a \Delta E_{E^{+}}$ & $a E_{T_{2}^{+}}$ & $a \Delta E_{T_{2}^{+}}$ \\
\hline A12 & $0.3046(53)$ & $0.031(14)$ & $0.3007(54)$ & $0.027(15)$ & $0.3076(66)$ & $0.034(15)$ \\
B12 & $0.242(15)$ & $-0.028(17)$ & $0.2679(48)$ & $-0.0013(93)$ & $0.245(10)$ & $-0.025(13)$ \\
C12 & $0.2136(71)$ & $-0.024(10)$ & $0.2241(52)$ & $-0.0187(98)$ & $0.2503(39)$ & $0.0002(77)$ \\
D12 & $0.1905(74)$ & $-0.043(11)$ & $0.2096(58)$ & $-0.0236(99)$ & $0.2231(41)$ & $-0.0100(83)$ \\
E12 & $0.1641(87)$ & $-0.019(10)$ & $0.174(11)$ & $-0.026(12)$ & $0.1457(86)$ & $-0.0400(90)$ \\
A16 & $0.3010(17)$ & $0.0079(36)$ & $0.3031(20)$ & $0.0100(37)$ & $0.3022(18)$ & $0.0091(36)$ \\
\hline \hline
\end{tabular}

TABLE XXI. One-particle mass in the multiple representations with $L=16$ using the continuum dispersion relation. An empty slot (...) for $a m_{V}$ indicates that the determination has not been possible due to the precision. The last column is the expected energy with the rest frame mass.

\begin{tabular}{lcllc}
\hline \hline $\mathbf{n}$ & $\Gamma$ & $a E$ & $a m_{V}$ & $\sqrt{\left(a m_{V}\right)^{2}+(a \mathbf{p})^{2}}$ \\
\hline$(0,0,0)$ & $T_{1}^{-}$ & $0.1466(16)$ & $0.1466(16)$ & $0.1466(16)$ \\
$(0,0,1)$ & $A_{1}$ & $0.4176(26)$ & $0.1420(77)$ & $0.4192(6)$ \\
& $E$ & $0.41797(80)$ & $0.1431(24)$ & $0.139(11)$ \\
$(1,1,0)$ & $A_{1}$ & $0.5724(27)$ & $0.129(12)$ & $0.5744(4)$ \\
& $B_{1}$ & $0.5702(28)$ & $0.1257(45)$ & $0.6958(6)$ \\
$(1,1,1)$ & $B_{2}$ & $0.5694(10)$ & $0.130(14)$ & $0.120(17)$ \\
$(0,0,2)$ & $A_{1}$ & $0.6925(27)$ & $\ldots$ & $0.7990(3)$ \\
\hline \hline
\end{tabular}


TABLE XXII. Energy for two particles in the moving frame with $d=(0,0,1)$.

\begin{tabular}{|c|c|c|c|c|c|c|}
\hline$\Gamma$ & $a E$ & $a \Delta E$ & $a E_{C M}$ & $\gamma$ & $a k$ & $a q$ \\
\hline$A_{1}$ & $0.5696(18)$ & $0.0038(28)$ & $0.4126(25)$ & $1.3805(40)$ & $0.1452(24)$ & $0.3698(61)$ \\
\hline$A_{2}$ & $0.5614(13)$ & $-0.0044(25)$ & $0.4012(18)$ & $1.3993(31)$ & $0.1369(21)$ & $0.3487(55)$ \\
\hline$B_{1}$ & $0.5674(19)$ & $0.0016(28)$ & $0.4096(26)$ & $1.3853(42)$ & $0.1430(25)$ & $0.3642(63)$ \\
\hline$B_{2}$ & $0.5666(11)$ & $0.0008(23)$ & $0.4084(16)$ & $1.3874(23)$ & $0.1422(20)$ & $0.3620(51)$ \\
\hline$E$ & $0.5835(14)$ & $0.0177(26)$ & $0.4316(18)$ & $1.3523(24)$ & $0.1583(35)$ & $0.4031(89)$ \\
\hline
\end{tabular}

TABLE XXIII. Energy spectrum in the $A_{1}$ representation.

\begin{tabular}{ccccc}
\hline \hline$L$ & Frame & $a E$ & $a k$ & $\delta_{0^{+}}\left({ }^{o}\right)$ \\
\hline 12 & $(0,0,0)+(0,0,0)$ & $0.3046(53)$ & $0.0582(82)$ & $-2.09(52)$ \\
& $(0,0,0)+(0,0,1)$ & $0.6840(40)$ & $0.1724(68)$ & $-0.9(1.2)$ \\
& $(0,0,0)+(0,0,0)$ & $0.3010(17)$ & $0.034(64)$ & $-1.41(75)$ \\
& $(0,0,1)+(0,0,1)$ & $0.8414(14)$ & $0.036(11)$ & $-0.80(72)$ \\
& $(0,0,0)+(0,0,1)$ & $0.5696(18)$ & $0.1452(24)$ & $-1.13(76)$ \\
& $(0,0,0)+(1,1,0)$ & $0.7176(14)$ & $0.1736(20)$ & $20.3(2.1)$ \\
& $(0,0,0)+(0,0,2)$ & $0.9127(19)$ & $0.1804(27)$ & $12.7(4.0)$ \\
\hline \hline
\end{tabular}

TABLE XXIV. Energy spectrum in the $A_{2}$ representation.

\begin{tabular}{lcccc}
\hline \hline$L$ & Frame & $a E$ & $a k$ & $\delta_{0^{-}}\left({ }^{o}\right)$ \\
\hline 16 & $(0,0,0)+(0,0,1)$ & $0.5614(13)$ & $0.1369(21)$ & $1.2(7)$ \\
& $(0,0,0)+(1,1,0)$ & $0.7077(16)$ & $0.1631(23)$ & $4.9(9)$ \\
& $(0,0,0)+(0,0,2)$ & $0.9101(18)$ & $0.1770(25)$ & $23(2)$ \\
\hline \hline
\end{tabular}

TABLE XXV. Obtained values for the phase shift $\delta_{1^{ \pm}}$in moving frame $(0,0,0)+(0,0,1)$ with the assumption of no mixings.

\begin{tabular}{lccc}
\hline \hline$\Gamma$ & $a k$ & Formula & $\delta\left(\left(^{\circ}\right)\right.$ \\
\hline$A_{1}$ & $0.1452(24)$ & $\cot \delta_{1^{-}}=\omega_{00}-\omega_{20}$ & $-0.14(10)$ \\
$A_{2}$ & $0.1369(21)$ & $\cot \delta_{1^{+}}=\omega_{00}-\omega_{20}$ & $0.127(60)$ \\
\hline \hline
\end{tabular}




\section{APPENDIX E: EXAMPLES OF $\mathcal{M}^{\Gamma}$ IN THE REST FRAME}

For the irreducible representations $E^{+}$and $T_{2}^{+}$, with the notation:

$$
\mathcal{M}_{J^{\prime} l^{\prime}, J l}^{T^{+} / E^{+}}=\left(\begin{array}{cc}
\left(\mathcal{M}_{22,22}^{T_{2}^{+} / E^{+}}\right)_{S=0} & 0 \\
0 & \left(\mathcal{M}_{J^{\prime} l^{\prime}, J l}^{T^{+} / E^{+}}\right)_{S=2}
\end{array}\right),
$$

with

$$
\begin{gathered}
\left(\mathcal{M}_{J^{\prime} l^{\prime}, J l}^{T^{+} / E^{+}}\right)_{S=2}=\left(\begin{array}{ccc}
\mathcal{M}_{20,20} & \mathcal{M}_{20,22} & \mathcal{M}_{20,24} \\
\mathcal{M}_{22,20} & \mathcal{M}_{22,22} & \mathcal{M}_{22,24} \\
\mathcal{M}_{24,20} & \mathcal{M}_{24,22} & \mathcal{M}_{24,24}
\end{array}\right) . \\
\mathcal{M}^{E^{+}}=\left(\begin{array}{cccc}
\omega_{00}+\frac{18}{7} \omega_{40} & 0 & 0 & 0 \\
0 & \omega_{00} & 0 & 3 \sqrt{\frac{2}{7}} \omega_{40} \\
0 & 0 & \omega_{00}+\frac{36}{49} \omega_{40} & \frac{30 \sqrt{5}}{49} \omega_{40} \\
0 & 3 \sqrt{\frac{2}{7}} \omega_{40} & \frac{30 \sqrt{5}}{49} \omega_{40} & \omega_{00}+\frac{27}{49} \omega_{40}
\end{array}\right), \\
\mathcal{M}_{2}^{T^{+}}=\left(\begin{array}{cccc}
\omega_{00}-\frac{12}{7} \omega_{40} & 0 & 0 & 0 \\
0 & \omega_{00} & 0 & -\frac{4}{\sqrt{14}} \omega_{40} \\
0 & 0 & \omega_{00}-\frac{24}{49} \omega_{40} & -\frac{20 \sqrt{5}}{49} \omega_{40} \\
0 & -\frac{4}{\sqrt{14}} \omega_{40} & -\frac{20 \sqrt{5}}{49} \omega_{40} & \omega_{00}-\frac{18}{49} \omega_{40}
\end{array}\right) .
\end{gathered}
$$

For the representation $A_{1}$ with the notation

$$
\begin{gathered}
\mathcal{M}_{J^{\prime} l^{\prime}, J l}^{A_{1}}=\left(\begin{array}{cc}
\mathcal{M}_{S=0}^{A_{1}} & 0 \\
0 & \mathcal{M}_{S=2}^{A_{1}}
\end{array}\right), \\
\left(\mathcal{M}_{J^{\prime} l^{\prime}, J l}^{A_{1}}\right)_{S=0}=\left(\begin{array}{lll}
\mathcal{M}_{00,00} & \mathcal{M}_{00,44} \\
\mathcal{M}_{44,00} & \mathcal{M}_{44,44}
\end{array}\right), \\
\left(\mathcal{M}_{J^{\prime} l^{\prime}, J l}^{A_{1}}\right)_{S=2}=\left(\begin{array}{llll}
\mathcal{M}_{02,02} & \mathcal{M}_{02,42} & \mathcal{M}_{02,44} & \mathcal{M}_{02,46} \\
\mathcal{M}_{42,02} & \mathcal{M}_{42,42} & \mathcal{M}_{42,44} & \mathcal{M}_{42,46} \\
\mathcal{M}_{44,02} & \mathcal{M}_{44,42} & \mathcal{M}_{44,44} & \mathcal{M}_{44,46} \\
\mathcal{M}_{46,02} & \mathcal{M}_{46,42} & \mathcal{M}_{46,44} & \mathcal{M}_{46,46}
\end{array}\right), \\
\left(\mathcal{M}_{J^{\prime} l^{\prime}, J l}^{A_{1}}\right)_{S=0}=\left(\begin{array}{cccc}
\omega_{00} & 6 \sqrt{\frac{3}{7}} \omega_{40} \\
6 \sqrt{\frac{3}{7}} \omega_{40} & \omega_{00}+\frac{4}{143}\left(81 \omega_{40}+260 \omega_{60}+140 \omega_{80}\right)
\end{array}\right) .
\end{gathered}
$$




$$
\begin{aligned}
& \left(\mathcal{M}_{J^{\prime} l^{\prime}, J l}^{A_{1}}\right)_{S=2} \\
& =\left(\begin{array}{cccc}
\omega_{00} & \frac{6 \sqrt{6}}{7} \omega_{40} & \frac{12}{7} \sqrt{\frac{15}{11}} \omega_{40} & 6 \sqrt{\frac{15}{77}} \omega_{40} \\
\frac{6 \sqrt{6}}{7} \omega_{40} & \omega_{00}+\frac{6}{7} \omega_{40} & \frac{12}{77} \sqrt{\frac{10}{11}}\left(9 \omega_{40}+35 \omega_{60}\right) & \frac{2}{143} \sqrt{\frac{70}{11}}\left(3 \omega_{40}+52 \omega_{60}+88 \omega_{80}\right) \\
\frac{12}{7} \sqrt{\frac{15}{11}} \omega_{40} & \frac{12}{77} \sqrt{\frac{10}{11}}\left(9 \omega_{40}+35 \omega_{60}\right) & \omega_{00}-\frac{2\left(1863 \omega_{40}+20566 \omega_{60}-10976 \omega_{80}\right)}{11011} & \frac{12 \sqrt{7}\left(45 \omega_{40}+208 \omega_{60}+72 \omega_{80}\right)}{1573} \\
6 \sqrt{\frac{15}{77}} \omega_{40} \frac{2}{143} \sqrt{\frac{70}{11}}\left(3 \omega_{40}+52 \omega_{60}+88 \omega_{80}\right) & \frac{12 \sqrt{7}\left(45 \omega_{40}+208 \omega_{60}+72 \omega_{80}\right)}{1573} & \frac{1573 \omega_{00}+2352 \omega_{40}+4160 \omega_{60}+560 \omega_{80}}{1573}
\end{array}\right) .
\end{aligned}
$$

\section{APPENDIX F: EFFECTIVE MASS}
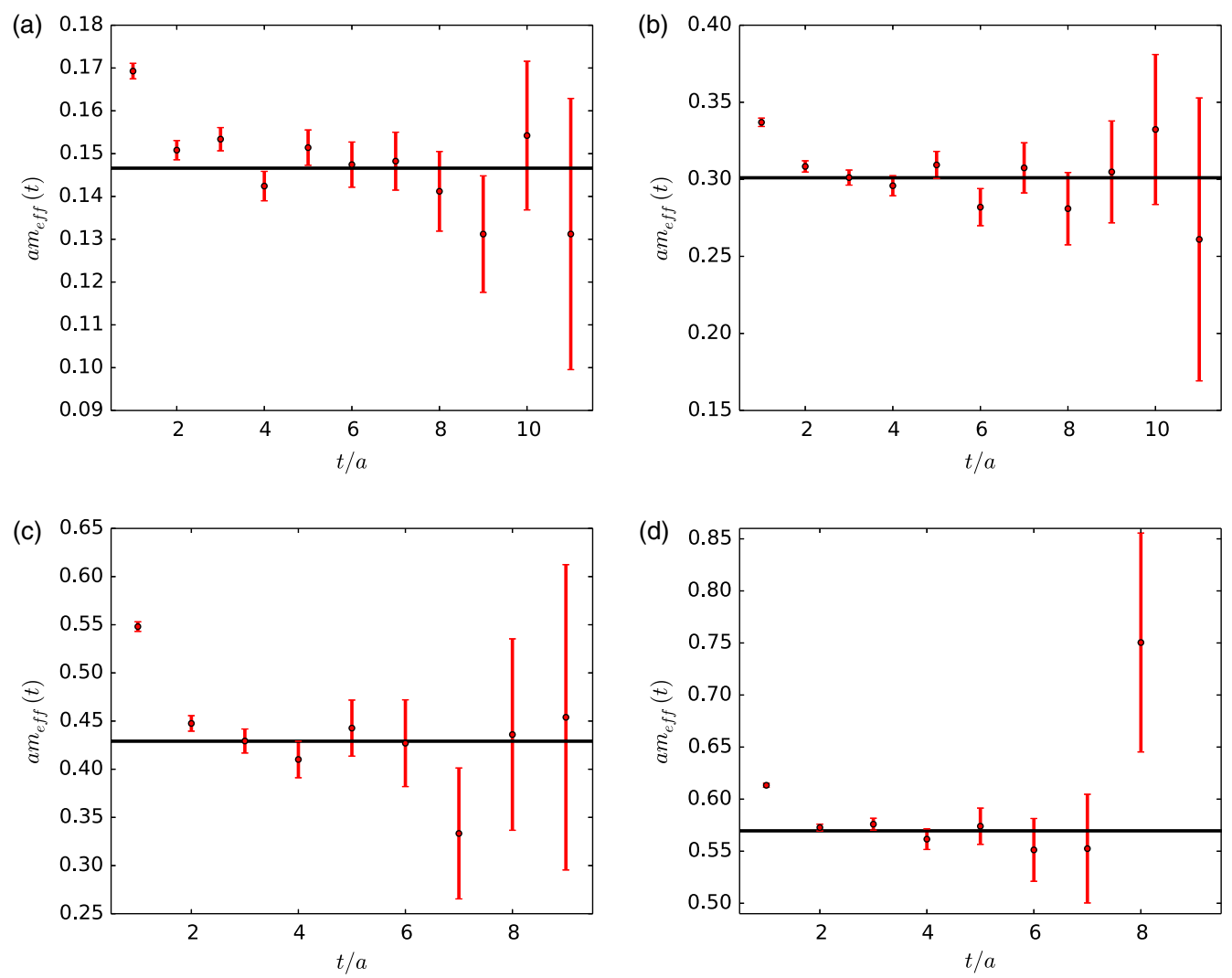

FIG. 4. Effective masses as a function of $t / a$ for ensemble A16. (a) Effective mass for the one-particle operator $\mathcal{O}^{T_{1}^{-}}$in the rest frame as in Table III. (b) Effective mass for the two-particle operator $\mathcal{O}^{A_{1}}$ in the rest frame as in Table V. (c) Effective mass for the one-particle operator $\mathcal{O}^{A_{1}}$ in the moving frame $d=(0,0,1)$ as in Table III. (d) Effective mass for the two-particle operator $\mathcal{O}^{A_{1}}$ in the moving frame $d=(0,0,0)+(0,0,1)$ as in Table V. 
[1] M. Lüscher, Nucl. Phys. B354, 531 (1991).

[2] K. Rummukainen and S. A. Gottlieb, Nucl. Phys. B450, 397 (1995).

[3] V. Bernard, M. Lage, U.-G. Meißner, and A. Rusetsky, J. High Energy Phys. 08 (2008) 024.

[4] R. A. Briceño, Z. Davoudi, and T. C. Luu, Phys. Rev. D 88, 034502 (2013).

[5] V. Bernard, D. Hoja, U. G. Meißner, and A. Rusetsky, J. High Energy Phys. 09 (2012) 023.

[6] Z. Fu, Phys. Rev. D 85, 014506 (2012).

[7] L. Leskovec and S. Prelovsek, Phys. Rev. D 85, 114507 (2012).

[8] M. Göckeler, R. Horsley, M. Lage, U.-G. Meißner, P. E. L. Rakow, A. Rusetsky, G. Schierholz, and J. M. Zanotti, Phys. Rev. D 86, 094513 (2012).

[9] R. A. Briceño, Phys. Rev. D 89, 074507 (2014).

[10] M. Lage, U.-G. Meißner, and A. Rusetsky, Phys. Lett. B 681, 439 (2009).

[11] C. Morningstar, J. Bulava, B. Singha, R. Brett, J. Fallica, A. Hanlon, and B. Hörz, Nucl. Phys. B924, 477 (2017).

[12] R. Frezzotti and G. C. Rossi, Phys. Rev. D 92, 054505 (2015).
[13] R. Frezzotti, M. Garofalo, and G. Rossi, Phys. Rev. D 93, 105030 (2016).

[14] S. Capitani et al., Eur. Phys. J. Web Conf. 175, 08009 (2018).

[15] S. Capitani et al., Eur. Phys. J. Web Conf. 175, 08008 (2018).

[16] F. Romero-López, C. Urbach, and A. Rusetsky, Eur. Phys. J. Web Conf. 175, 14013 (2018).

[17] J. Gasser, B. Kubis, and A. Rusetsky, Nucl. Phys. B850, 96 (2011).

[18] R. A. Briceño, Z. Davoudi, T. Luu, and M. J. Savage, Phys. Rev. D 88, 114507 (2013).

[19] H. A. Bethe, Phys. Rev. 76, 38 (1949).

[20] M. H. Ross and G. L. Shaw, Ann. Phys. (N.Y.) 13, 147 (1961).

[21] C. E. Thomas, R. G. Edwards, and J. J. Dudek, Phys. Rev. D 85, 014507 (2012).

[22] M. Lüscher, Nucl. Phys. B354, 531 (1991).

[23] H. Evertz, K. Jansen, J. Jersák, C. Lang, and T. Neuhaus, Nucl. Phys. B285, 590 (1987).

[24] K. Ottnad and C. Urbach (ETM Collaboration), Phys. Rev. D 97, 054508 (2018). 